\title{
Simple lumped circuit model applied to field flatness tuning of four-rod radio frequency quadrupoles
}

\author{
C. Y. Tan \\ Fermi National Accelerator Laboratory, P.O. Box 500, Batavia, Illinois 60510-5011, USA
}

J. S. Schmidt, and A. Schempp

IAP, Goethe University, Frankfurt am Main, Germany

(Received 26 April 2013; published 29 January 2014)

\begin{abstract}
The field flatness of any radio frequency quadrupole (RFQ) is an important parameter that needs to be carefully tuned because it can affect beam transmission efficiency. In four-rod RFQs, the heights of a set of tuning plates determine the quality of the field flatness. The goals of this paper are (a) to show that by using a lumped circuit model of a four-rod RFQ, the field flatness profile for any tuning plate height distribution can be quickly calculated, (b) to derive a perturbative solution of the model so that insights into the physics of the tuning process and its effects can be understood, and (c) to compare the predicted field profiles to measurements.
\end{abstract}

\section{INTRODUCTION}

The field flatness (see Sec. IIA1) of any radio frequency quadrupole (RFQ) is an important parameter that needs to be well controlled because it can affect beam transmission efficiency [1]. In the four-rod RFQ shown in Fig. 1, tuning plates are used to change the voltage in each tuning cell by raising or lowering them. The goal is to move each plate until the field flatness is found that is within some prescribed tolerance, usually $<5 \%$. However, moving the tuning plates also changes the resonant frequency of the RFQ and so the entire process can be quite tedious when this constraint is also included.

Historically, the tuning process is done by moving the tuning plates of the RFQ using an iterative trial and error process that can be grossly inefficient [1]. Attempts have been made to semiautomate this process by precalculating the tuning plate heights with CST Microwave STUdiO ${ }^{\circledR}$ (MWS). This simulation tool has been used successfully in the design and tuning for various types of rf accelerators including drift-tube linacs as well as four-vane RFQs. However, the agreement between simulation and measurement can be rather poor for four-rod RFQs [2-5]. An explanation for that could be that the simulation accuracy of MWS is quite different for transmission-line resonators compared to cavity resonators. For example, Fig. 2 presents the results of the MWS field flatness

\footnotetext{
*ytan@fnal.gov

Published by the American Physical Society under the terms of the Creative Commons Attribution 3.0 License. Further distribution of this work must maintain attribution to the author(s) and the published articles title, journal citation, and DOI.
}

simulation for a fully imported CAD model of the Fermi National Accelerator Laboratory (FNAL) RFQ. We show here the results of five models with different meshes that have been refined and varied to improve the simulation accuracy. All of these models have several million mesh cells and all of them exhibit a slope in the simulated field distribution from the low energy end to the high energy end that is not present in our measurement. The deviation between the simulated and measured field flatness in these models can be up to $14 \%$. Similar results have been presented by Kurennoy [5] for the same RFQ. To overcome this problem for particle tracking simulations, a fictive tuning setup that is not the one used in the RFQ

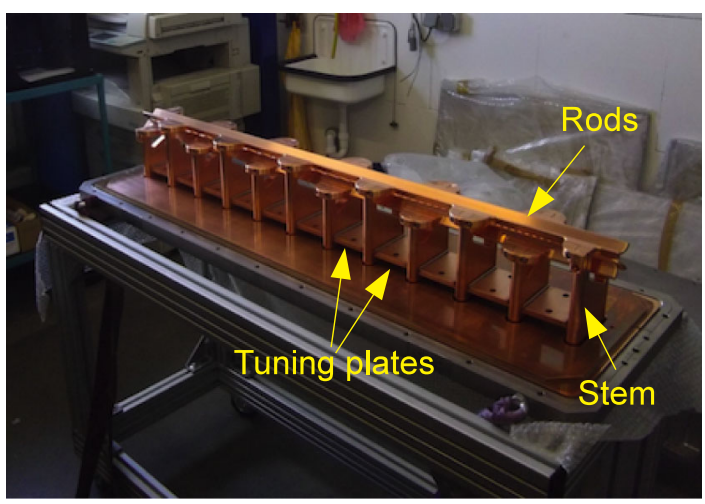

FIG. 1 (color online). This picture shows the FNAL injector RFQ outside of its vacuum tank. The four rods are held by stems and the tuning plates are located between stems. There are 13 tuning cells in this RFQ with 11 full cells and a half cell at each end (for a total of two half cells). The beginning and end cell tuning plates are not installed here. When installed, they go between the vacuum tank wall and the first and last stem. 


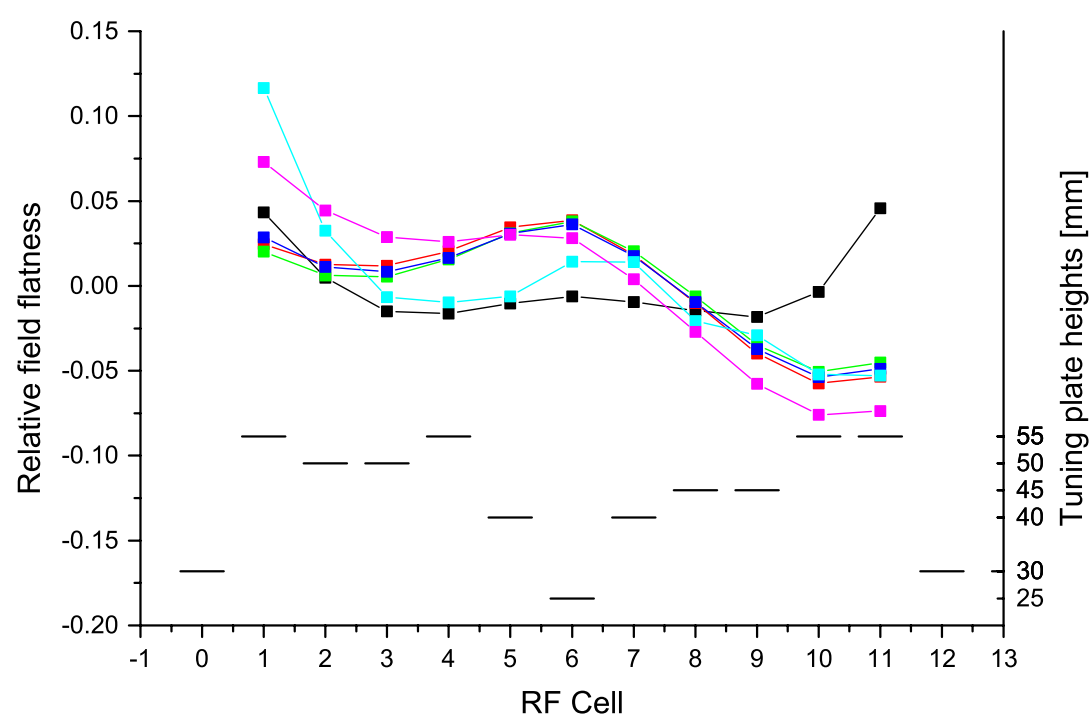

-m-Measurement

FIG. 2 (color online). Comparison of a measured field flatness with its MWS simulation. In this figure, a measured field flatness of the FNAL RFQ (black squares) for a given tuning plate distribution (-) is compared to the simulation of the fully imported model of this RFQ that has the same tuning setup (Mod1 to Mod5). The simulation models differ in their meshing which has been refined in several steps. All of the simulated field flatness results show a slope from the low energy to the high energy end that is not present in the measurement. The simulated values can deviate up to $14 \%$ from the measured value.

is used to reproduce the measured field flatness in simulations. With this field flatness, reasonable particle dynamic simulations can be performed, for example, discussed in Ref. [5].

Coming back to tuning the RFQ, the lack of an accurate real-time model for the tuning process results in the reversion back to the traditional iterative method.

It is with the above problems in mind that we want to see whether a lumped circuit model of the four-rod RFQ can be used instead. After we developed this model, we found that the lumped circuit model has at least two advantages: the first is that the solution to the problem essentially becomes an eigenvalue problem that is easily and quickly solved both analytically and numerically. The second is that the fundamental physical relations of the resonator system become more apparent when compared to the 3D results of detailed models used for MWS simulations.

The idea of modeling a four-rod RFQ with a lumped circuit model is not new. For example see Refs. [6] and [7] or [8] for its application on a four-vane RFQ. In this paper, we will use a simplified model for four-rod RFQs first published by Fang et al. in 1992 [9] which we will improve and expand upon.

\section{THEORY}

The motivation behind modeling the RFQ as a series of coupled $L C$ circuits comes from the observation that each tuning cell can be modeled as an $L C$ equivalent circuit. From Fig. 3, we can see that, since there are gaps between the rods, they are capacitively coupled. The stems and the base together allow a current to flow in a loop and thus this structure can be thought of as an inductor. Together, the rods, stems, and base form the basic $L C$ circuit for each tuning cell. In order to change
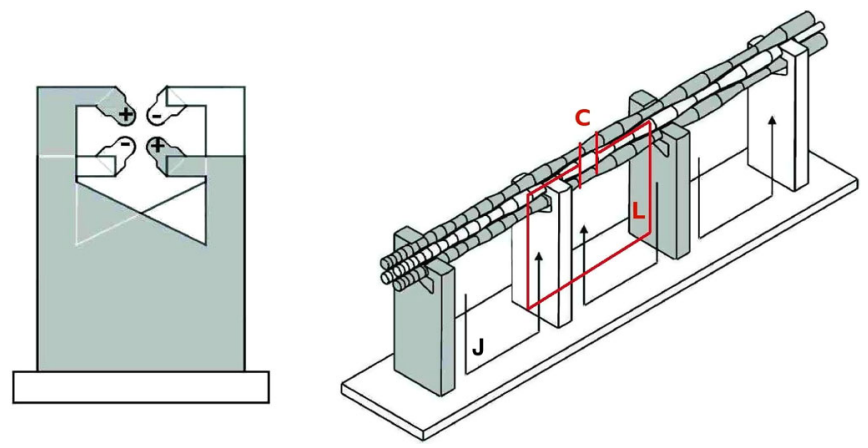

FIG. 3 (color online). This is a cartoon of a four-rod RFQ that has three tuning cells. Each tuning cell can be modeled as an $L C$ circuit with a current $J$ flowing in each cell. Notice that the stems alternatively hold two rods, i.e. the shaded stems hold the shaded rods, while the unshaded stems hold the unshaded rods. The end view of the rod-stem setup shows the quadrupole voltage polarity structure that arises when the currents flow in opposite directions in adjacent tuning cells. This is the necessary quadrupole polarity structure that is required for transverse focusing. 
the resonant frequency of a cell, a tuning plate is inserted into that cell so that the current path is shortened which, in effect, lowers the inductance of that cell.

Of course, each $L C$ circuit does not live in isolation. In fact, according to Fang, they are coupled not only to their nearest neighbor but also to their next nearest neighbor. With this in mind, we can create the lumped circuit model of the RFQ shown in Fig. 4. It is important to take into account the direction of the winding of the inductor represented by black dots in the model shown in Fig. 4 . In this model, the tuning cells are numbered from $0, \ldots, N$ with cell 0 and $N$ being the half cells. Each tuning cell has capacitance $C_{n}$ for $n=0, \ldots \ldots, N$ and $L_{n}$ for $n=1, \ldots \ldots, N-1, L_{0} / 2$ for cell 0 , and $L_{N} / 2$ for cell $N$. The coupling between the nearest neighbor cells is $2 \kappa_{1}$ and the next nearest neighbor cells is $2 \kappa_{2}$. It will be seen later when we compare our model to the MWS results, that it is sufficient to have $\kappa_{1}$ and $\kappa_{2}$ independent of cell number [10]. Finally, a current $J_{n}$ for $n=0, \ldots . ., N$ flows in each loop.

Let us examine cell 0 first. We can write down Kirchhoff's equation for it and it is

$$
\begin{array}{r}
\frac{J_{0}}{i \omega C_{0}}+\frac{1}{2} i \omega L_{0} J_{0}-i \kappa_{1} \omega \sqrt{L_{0} L_{1}} J_{1}-i \kappa_{2} \omega \sqrt{L_{0} L_{2}} J_{2}=0 \\
\Rightarrow-J_{0}\left(1-\frac{1}{2} \omega^{2} C_{0} L_{0}\right)-\kappa_{1} \omega^{2} \sqrt{L_{0} L_{1}} C_{0} J_{1}-\kappa_{2} \omega^{2} \sqrt{L_{0} L_{1}} C_{0} J_{2}=0 .
\end{array}
$$

Similarly, we can write down Kirchhoff's equation for the last cell, $N$, and it is

$$
-\kappa_{2} \omega^{2} \sqrt{L_{N-2} L_{N}} C_{N} J_{N-2}-\kappa_{1} \omega^{2} \sqrt{L_{N-1} L_{N}} C_{N} J_{N-1}-J_{N}\left(1-\frac{1}{2} \omega^{2} C_{N} L_{N}\right)=0 .
$$

For any arbitrary cell, $n$, we have

$$
\begin{aligned}
& -\kappa_{2} \omega^{2} \sqrt{L_{n-2} L_{n}} C_{n} J_{n-2}-\kappa_{1} \omega^{2} \sqrt{L_{n-1} L_{n}} C_{n} J_{n-1} \\
& \quad-J_{n}\left(1-\omega^{2} L_{n} C_{n}\right)-\kappa_{1} \omega^{2} \sqrt{L_{n} L_{n+1}} C_{n} J_{n+1}-\kappa_{2} \omega^{2} \sqrt{L_{n} L_{n+2}} C_{n} J_{n+2}=0
\end{aligned}
$$

for $n=1, \ldots \ldots, N-1$. If any subscript is less than zero or greater than $N$, the term is identically zero. For example, $L_{-1}=J_{-1}=0$.

\section{A. Eigensystem equation}

We can combine Eqs. (1)-(3) and turn them into an eigenvector and eigenvalue problem

$$
\left(\boldsymbol{U}_{1}-\lambda^{2} \boldsymbol{I}\right) \boldsymbol{J}=0
$$

where $\lambda^{2}=1 / \omega^{2}$,

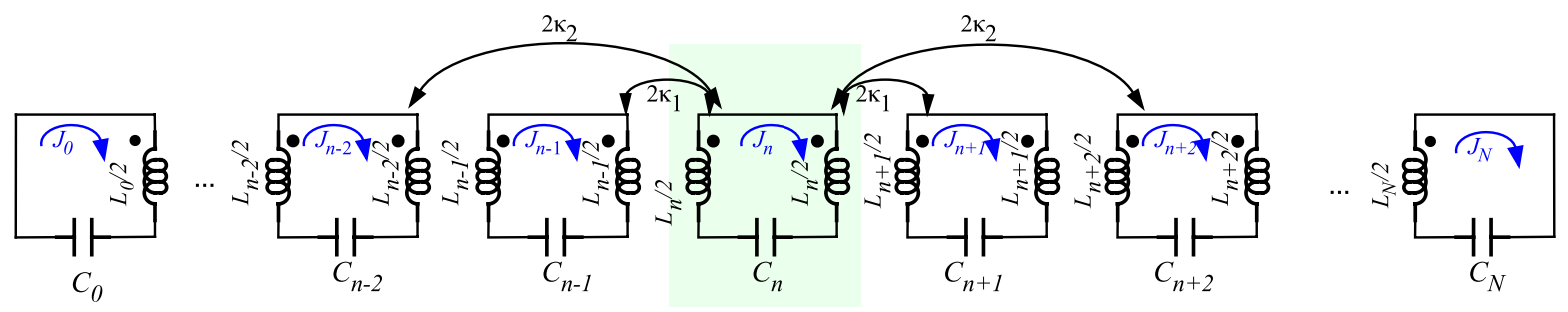

FIG. 4 (color online). The RFQ lumped circuit model. The highlighted $n$th cell shows how it is coupled to its first and second neighbor circuits. Each closed circuit represents a tuning cell. Notice the filled dot that is associated with each inductor. The location of the filled dot and the direction of the current determines the sign of the induced voltage that comes from coupling between cells. For an example, see $[11]$. 


$$
\boldsymbol{U}_{1}=\left(\begin{array}{ccccccc}
m_{0,0} C_{0} & m_{0,1} C_{0} & m_{0,2} C_{0} & 0 & \ldots & \ldots & 0 \\
m_{0,1} C_{1} & m_{1,1} C_{1} & m_{1,2} C_{1} & m_{1,3} C_{1} & \ddots & & \vdots \\
m_{0,2} C_{2} & m_{1,2} C_{2} & \ddots & \ddots & \ddots & \ddots & \vdots \\
0 & m_{1,3} C_{3} & \ddots & \ddots & \ddots & m_{N-3, N-1} C_{N-3} & 0 \\
\vdots & \ddots & \ddots & \ddots & \ddots & m_{N-2, N-1} C_{N-2} & m_{N-2, N} C_{N-2} \\
\vdots & & \ddots & m_{N-3, N-1} C_{N-1} & m_{N-2, N-1} C_{N-1} & m_{N-1, N-1} C_{N-1} & m_{N-1, N} C_{N-1} \\
0 & \ldots & \cdots & 0 & m_{N-2, N} C_{N} & m_{N-1, N} C_{N} & m_{N, N} C_{N}
\end{array}\right)
$$

is a band matrix with

$$
\left.\begin{array}{l}
m_{0,0}=\frac{1}{2} L_{0}, \quad m_{N, N}=\frac{1}{2} L_{N} \quad m_{n, n}=L_{n} \\
m_{n-1, n}=-\kappa_{1} \sqrt{L_{n-1} L_{n}} \quad m_{n-2, n}=-\kappa_{2} \sqrt{L_{n-2} L_{n}}
\end{array}\right\} \quad \text { for } n=1, \ldots, N-1
$$

and

$$
\boldsymbol{J}=\left(\begin{array}{c}
J_{0} \\
J_{1} \\
J_{2} \\
\vdots \\
J_{N}
\end{array}\right), \quad \boldsymbol{I}=\left(\begin{array}{ccccc}
1 & 0 & \ldots & \ldots & 0 \\
0 & \ddots & \ddots & & \vdots \\
\vdots & \ddots & \ddots & \ddots & \vdots \\
\vdots & & \ddots & \ddots & 0 \\
0 & \ldots & \ldots & 0 & 1
\end{array}\right)
$$

It is obvious that if the coupling constants $\kappa_{1,2}$, capacitances $C_{0, \ldots \ldots, N}$, and inductances $L_{0, \ldots \ldots, N}$ are specified, we can numerically solve Eq. (4) for the eigenmodes and eigenvectors of $\boldsymbol{U}_{1}$. Note, it is easy to show that the eigenmodes of $\lambda^{2}$ are the same as $1 / \lambda^{2}$.

\section{Definition of field flatness}

We define the voltage $V_{n}$ in tuning cell $n$ to be

$$
V_{n}=\frac{J_{n}}{\Omega C_{n}} \quad n=0, \ldots, N,
$$

where $\Omega$ is the resonant frequency of the RFQ. Notice that we have left out the $i$ in Eq. (8) because the relative phase between current and voltage is irrelevant in this application.

We define the relative field flatness of tuning cell $n$ to be

$$
\epsilon_{n}=\frac{\left|V_{n}\right|-\langle|V|\rangle}{\langle|V|\rangle}
$$

where $\langle|V|\rangle$ is the mean of the absolute voltage in the tuning cells from $n=1, \ldots ., N-1$. Notice that we have left out the end cells in the calculation of the mean because the voltages in these cells tend to be very far from the mean because they are dominated by boundary conditions. By extension, we define the "normalized" voltage in tuning cell $n$ to be

$$
\text { normalized } V_{n}=\frac{\left|V_{n}\right|}{\sqrt{\sum_{m=1}^{N-1}\left|V_{m}\right|^{2}}}
$$

The field flatness of the RFQ is defined to be the difference between the maximum and minimum relative field flatness found in the list $\left\{\epsilon_{n} \mid n=1, \ldots \ldots, N-1\right\}$. The goal is to "flatten" this field, i.e. to make the difference as small as possible. An example of a field profile and field flatness error is shown in Fig. 5. This example has been calculated

\begin{tabular}{|c|c|c|c|}
\hline Parameter & Value & Units & Description \\
\hline$N+1$ & 13 & & Number of tuning cells \\
\hline$C$ & 8.3 & $\mathrm{pF}$ & $\begin{array}{c}C_{n}=C \text { for } n=1, \ldots ., N-1, \\
C_{0}=C_{N}=2 C\end{array}$ \\
\hline$L$ & 0.108 & $\mu \mathrm{H}$ & $L_{n}=L$ for $n=0, \ldots \ldots, N$ \\
\hline$\kappa_{1}$ & 0.0936 & & First neighbor coupling \\
\hline$\kappa_{2}$ & 0.0031 & & Second neighbor coupling \\
\hline$a$ & $-1.00 \times 10^{-6}$ & $\mathrm{~mm}^{-2}$ & Coefficient of $h^{2}$ in $\delta L(h) / L$ \\
\hline$b$ & $-0.38 \times 10^{-4}$ & $\mathrm{~mm}^{-1}$ & Coefficient of $h$ in $\delta L(h) / L$ \\
\hline
\end{tabular}
using the parameters shown in Table I, for an ideal four-rod RFQ that has no flaws resulting from manufacture. The curvature that we see here, which we will discuss in Sec. IIIB2, is due to $\kappa_{2} \neq 0$. So, even in a perfectly

TABLE I. Lumped circuit model parameters. 

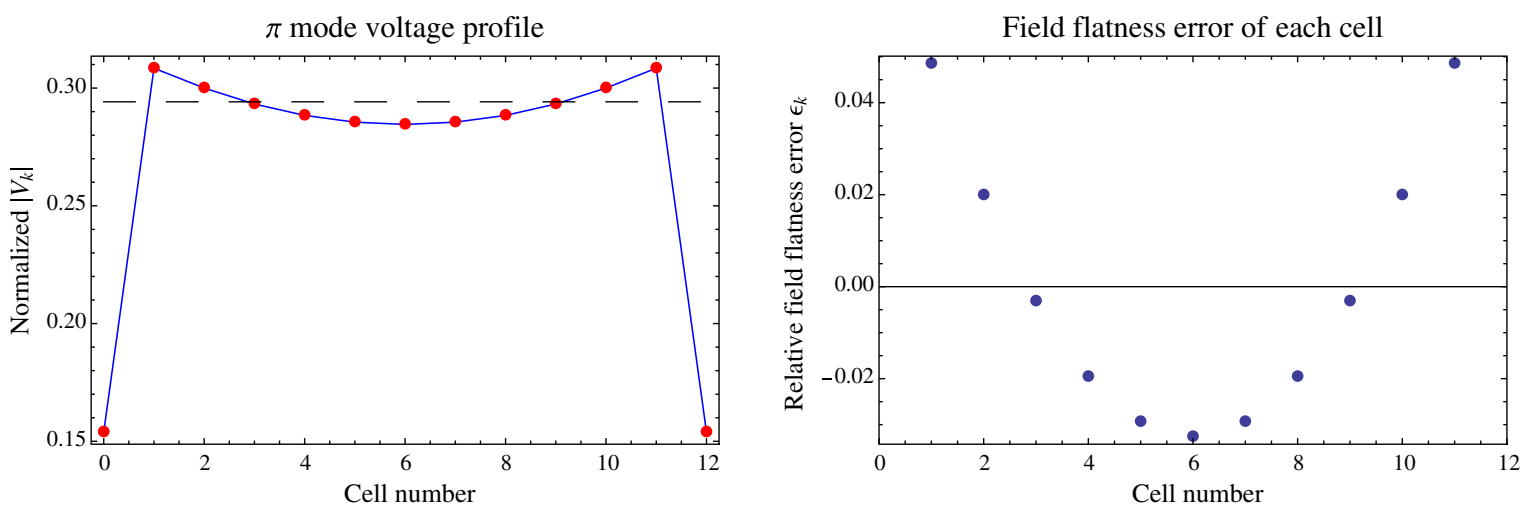

FIG. 5 (color online). This is an example of a field profile and error. The dashed line in the graph on the left is $\langle|V|\rangle$ which was defined in Sec. IIA1. In this example, the field error is $8 \%$.

manufactured four-rod RFQ, we may still have to flatten the fields because the field error can be $>5 \%$.

\section{B. Special case}

In principle, once we are armed with the eigenvalue equation of the previous section, we can simply fill in the required parameters and numerically calculate the eigenmodes and eigenvectors. However, this defeats one purpose of using the lumped circuit model that is to give us insights into the physics of the tuning process.

The first step in our quest for understanding is to simplify the system. We will set the resonant frequency of each cell to be the same. Thus, if we set $L_{n}=L$ for $n=0, \ldots ., N$, then it is obvious from Fig. 4 that we must have $C_{n}=C$ for $n=1, \ldots ., N-1$, and $C_{0}=C_{N}=2 C$. Thus, the $\boldsymbol{U}_{1}$ matrix becomes

$$
\boldsymbol{U}_{1}=\lambda_{c}^{2}\left(\begin{array}{ccccccccc}
1 & -2 \kappa_{1} & -2 \kappa_{2} & 0 & \cdots & \cdots & \cdots & \cdots & 0 \\
-\kappa_{1} & 1 & -\kappa_{1} & -\kappa_{2} & \ddots & & & & \vdots \\
-\kappa_{2} & -\kappa_{1} & 1 & -\kappa_{1} & -\kappa_{2} & \ddots & & & \vdots \\
0 & -\kappa_{2} & -\kappa_{1} & 1 & -\kappa_{1} & -\kappa_{2} & \ddots & & \vdots \\
\vdots & \ddots & \ddots & \ddots & \ddots & \ddots & \ddots & \ddots & \vdots \\
\vdots & & \ddots & \ddots & \ddots & \ddots & -\kappa_{1} & -\kappa_{2} & 0 \\
\vdots & & & \ddots & \ddots & \ddots & 1 & -\kappa_{1} & -\kappa_{2} \\
\vdots & & & & \ddots & \ddots & -\kappa_{1} & 1 & -\kappa_{1} \\
0 & \cdots & \cdots & \ldots & \cdots & 0 & -2 \kappa_{2} & -2 \kappa_{1} & 1
\end{array}\right) \equiv \lambda_{c}^{2} \boldsymbol{U}_{0}\left(\kappa_{1}, \kappa_{2}\right)
$$

where $\lambda_{c}^{2}=1 / \omega_{c}^{2}=L C$. Clearly, $\boldsymbol{U}_{1}$ is a band matrix and it is not pentadiagonal or symmetric because of the first and last rows.

Next, we set $\kappa_{2}=0$ to see and find analytic solutions for the eigenvalues and eigenvectors of $\boldsymbol{U}_{0}\left(\kappa_{1}, 0\right)$. Once we find them, we can apply perturbation theory to arrive at a solution for the case when $\kappa_{2} \ll \kappa_{1}$.

\section{Ansatz for finding the eigenvalues and eigenvectors of $U_{0}\left(\kappa_{1}, 0\right)$}

The clue to finding the eigenvalues and eigenvectors of $\boldsymbol{U}_{0}\left(\kappa_{1}, 0\right)$ is in its numerical solutions. An example is shown in Fig. 6. From this figure, it is obvious that we can propose an ansatz for the eigenvectors of $\boldsymbol{U}_{0}\left(\kappa_{1}, 0\right)$ to have the form 

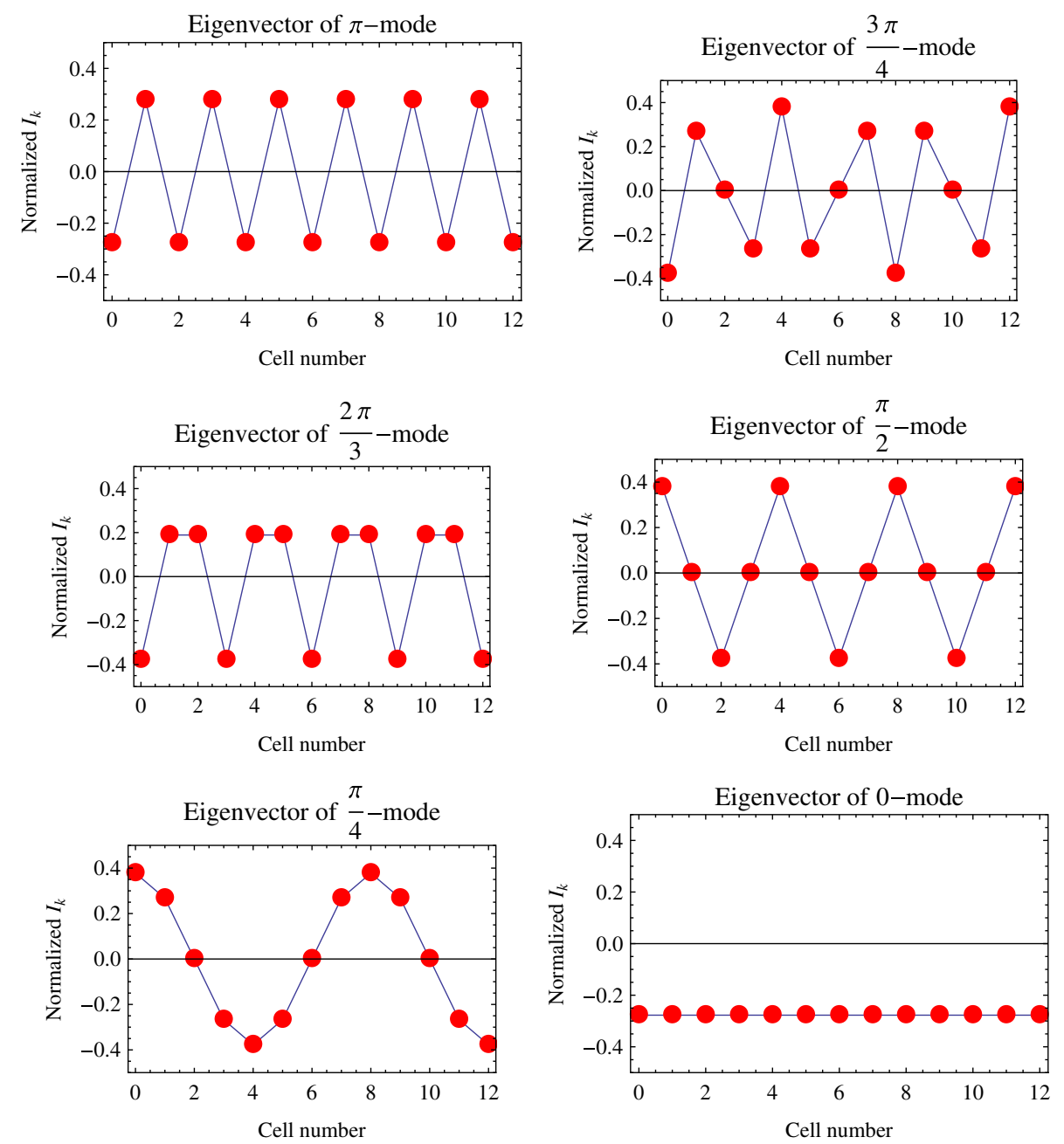

FIG. 6 (color online). The normalized eigenvectors of $\boldsymbol{U}_{0}\left(\kappa_{1}, 0\right)$ plotted in blue and the ansatz plotted as red filled dots for a few modes. It is clear that the ansatz matches the numerically calculated eigenvectors exactly.

$$
\boldsymbol{J}_{k}=A_{k}\left(\begin{array}{c}
\cos \frac{0 \times k \pi}{N} \\
\cos \frac{1 \times k \pi}{N} \\
\cos \frac{2 \times k \pi}{N} \\
\vdots \\
\cos \frac{(N-1) \times k \pi}{N} \\
\cos \frac{N \times k \pi}{N}
\end{array}\right) \quad \text { for } k=0, \ldots, N
$$

where $A_{k}=1 /\left\|\boldsymbol{J}_{k}\right\|$ and $\|\cdot\|$ is the norm of a vector. It is easy to show that

$$
\frac{1}{\left\|\boldsymbol{J}_{k}\right\|}= \begin{cases}\frac{1}{\sqrt{N / 2+1}} & \text { if } k \neq 0 \text { or } k \neq N \\ \frac{1}{\sqrt{N+1}} & \text { if } k=0 \text { or } k=N .\end{cases}
$$

Note, it is interesting that $\boldsymbol{J}_{k}$ is independent of $\kappa_{1}$.

Let us create a matrix from the column vectors $\boldsymbol{J}_{k}$ :

$$
\boldsymbol{J}=\left(\begin{array}{llll}
\boldsymbol{J}_{0} & \boldsymbol{J}_{1} & \cdots & \boldsymbol{J}_{N}
\end{array}\right)
$$

and after a bit of algebra, we can show that $\boldsymbol{J}_{k}$ are the eigenvectors of $\boldsymbol{U}_{1}\left(\kappa_{1}, 0\right)$ because

$$
\underline{J}^{-1} \boldsymbol{U}_{0}\left(\kappa_{1}, 0\right) \boldsymbol{J}=\left(\begin{array}{ccccc}
\Lambda_{0} & 0 & \cdots & \cdots & 0 \\
0 & \Lambda_{1} & \ddots & & \vdots \\
\vdots & \ddots & \ddots & \ddots & \vdots \\
\vdots & & \ddots & \ddots & 0 \\
0 & \cdots & \cdots & 0 & \Lambda_{N}
\end{array}\right)=\Lambda,
$$

where $\Lambda$ is a diagonal matrix whose entries $\left\{\Lambda_{k} \mid k=\right.$ $0, \ldots ., N\}$ are the eigenvalues of $\boldsymbol{U}_{0}\left(\kappa_{1}, 0\right)$ and

$$
\Lambda_{k}=1-2 \kappa_{1} \cos \frac{k \pi}{N} \quad \text { for } k=0, \ldots, N .
$$

Therefore, for $k=N$ which is the $\pi$ mode, we can select out the $N$ th column of $\boldsymbol{J}$ and its associated eigenvalue to get 


$$
\boldsymbol{J}_{N}=\frac{1}{\sqrt{N+1}}\left(\begin{array}{c}
+1 \\
-1 \\
+1 \\
\vdots \\
(-1)^{N}
\end{array}\right) \quad \Omega_{N}=\frac{\omega_{c}}{\sqrt{\Lambda_{N}}}=\frac{\omega_{c}}{\sqrt{1+2 \kappa_{1}}},
$$

where $\Omega_{N}$ is the resonant frequency of the $k=N$ mode.

\section{2. $\pi$ mode}

The reason why the $\pi$ mode is the required mode for four-rod RFQs is seen by looking at how the rods are mounted on the stems. It is apparent from Fig. 3 that two rods are held on the same stems that alternate with the stems that hold the other two rods. Therefore, for the rods to have a quadrupole voltage polarity structure, the nearest neighbor tuning cells must have opposite signs in voltage. Hence, the $\pi$ mode is necessary because it is clear from Eq. (17) that this requirement is satisfied when the currents flow in opposite directions in adjacent cells. MWS simulations shown in Appendix C confirm that the currents do flow in opposite directions between adjacent tuning cells for the operating mode which is the $\pi$ mode.

\section{Application of perturbation theory for finding the eigenvalues and eigenvectors of $U_{0}\left(\kappa_{1}, \kappa_{2}\right)$}

We will show how the eigenvalues and eigenvectors are affected by the introduction of the next nearest neighbor coupling $\kappa_{2}$. The first step is to partition $\boldsymbol{U}_{0}\left(\kappa_{1}, \kappa_{2}\right)$ into two parts

$$
\boldsymbol{U}_{0}\left(\kappa_{1}, \kappa_{2}\right)=\boldsymbol{U}_{0}\left(\kappa_{1}, 0\right)+\left(\begin{array}{ccccccccc}
0 & 0 & -2 \kappa_{2} & 0 & \cdots & \cdots & \cdots & \ldots & 0 \\
0 & 0 & 0 & -\kappa_{2} & \ddots & & & & \vdots \\
-\kappa_{2} & 0 & 0 & 0 & -\kappa_{2} & \ddots & & & \vdots \\
0 & -\kappa_{2} & 0 & 0 & 0 & -\kappa_{2} & \ddots & & \vdots \\
\vdots & \ddots & \ddots & \ddots & \ddots & \ddots & \ddots & \ddots & \vdots \\
\vdots & & \ddots & \ddots & \ddots & \ddots & 0 & -\kappa_{2} & 0 \\
\vdots & & & \ddots & \ddots & \ddots & 0 & 0 & -\kappa_{2} \\
\vdots & & & & \ddots & -\kappa_{2} & 0 & 0 & 0 \\
0 & \ldots & \ldots & \ldots & \ldots & 0 & -2 \kappa_{2} & 0 & 0
\end{array}\right) \equiv \boldsymbol{U}_{0}\left(\kappa_{1}, 0\right)+\delta \boldsymbol{U}_{0}\left(\kappa_{2}\right)
$$

for $\kappa_{2} \ll \kappa_{1}$. Again, we notice that the first and last rows of $\delta \boldsymbol{U}_{0}\left(\kappa_{2}\right)$ break its symmetry.

Since the equation that we are going to solve perturbatively is reminiscent of those studied in quantum mechanics (QM), we can, in fact, use the formal perturbation machinery developed in $\mathrm{QM}$ to solve our problem. So in that spirit, we will use the "bra" "ket" notation used in QM to write $\boldsymbol{J}_{k}$ as an eigenket with eigenvalue $\Lambda_{k}$ :

$$
\boldsymbol{J}_{k}=\left|\Lambda_{k}\right\rangle .
$$

However, unlike QM where only Hermitian operators are considered, $\boldsymbol{U}_{0}\left(\kappa_{1}, 0\right)$ is not symmetric and hence $\left\{\left|\Lambda_{k}\right\rangle \mid k=0, \ldots . ., N\right\}$ is not an orthonormal set. Fortunately, all is not lost, as long as we define the eigenbras $\left\{\left\langle\Lambda_{k}|| k=0, \ldots . ., N\right\}\right.$ properly we can salvage the perturbative technique. The artifice is shown in Appendix A where we find that

$$
\left\langle\Lambda_{k}\right|=\left(\boldsymbol{J}^{-1}\right)_{k},
$$

where $\left(\boldsymbol{J}^{-1}\right)_{k}$ is the $(k+1)$ th row of the matrix $\boldsymbol{J}^{-1}$. (Note, the exact form of both $\boldsymbol{J}$ and $\boldsymbol{J}^{-1}$ are shown in Appendix B.) Thus, we can have the orthonormality condition satisfied, i.e.

$$
\left\langle\Lambda_{k} \mid \Lambda_{\ell}\right\rangle=\delta_{k \ell},
$$

where $\delta_{k \ell}$ is the Kronecker $\delta$. Thus,

$$
\left\langle\Lambda_{k}\left|\boldsymbol{U}_{0}\left(\kappa_{1}, 0\right)\right| \Lambda_{k}\right\rangle=\Lambda_{k} .
$$

Let us assume that the eigenvector of $\boldsymbol{U}_{0}\left(\kappa_{1}, \kappa_{2}\right)$ is $\left|\Lambda_{k}^{\prime}\right\rangle$ and it can be divided into two parts:

$$
\left|\Lambda_{k}^{\prime}\right\rangle=\left|\Lambda_{k}\right\rangle+\left|\delta \Lambda_{k}\right\rangle
$$


so that

$$
\left.\begin{array}{rl}
\boldsymbol{U}_{0}\left(\kappa_{1}, \kappa_{2}\right)\left|\Lambda_{k}^{\prime}\right\rangle & =\left[\boldsymbol{U}_{0}\left(\kappa_{1}, 0\right)+\delta \boldsymbol{U}_{0}\left(\kappa_{2}\right)\right]\left[\left|\Lambda_{k}\right\rangle+\left|\delta \Lambda_{k}\right\rangle\right] \\
& =\left[\Lambda_{k}+\delta \Lambda_{k}\right]\left[\left|\Lambda_{k}\right\rangle+\left|\delta \Lambda_{k}\right\rangle\right] \\
+\delta \boldsymbol{U}_{0}\left(\kappa_{2}\right)\left|\Lambda_{k}\right\rangle & =\Lambda_{k}\left|\delta \Lambda_{k}\right\rangle+\delta \Lambda_{k}\left|\Lambda_{k}\right\rangle
\end{array}\right\}
$$

because in first order perturbation theory, we can neglect the terms $\delta \boldsymbol{U}_{0}\left(\kappa_{1}\right)\left|\delta \Lambda_{k}\right\rangle$ and $\delta \Lambda_{k}\left|\delta \Lambda_{k}\right\rangle$. We will assume that $\left\{\left|\Lambda_{k}\right\rangle \mid k=0, \ldots \ldots, N\right\}$ forms a complete set so that we can write

$$
\left|\delta \Lambda_{k}\right\rangle=\sum_{\ell=0}^{N} c_{k \ell}\left|\Lambda_{\ell}\right\rangle,
$$

where $c_{k \ell}$ are constants to be determined.

When we substitute Eq. (25) into Eq. (24), we have

$$
\begin{gathered}
\sum_{\ell=0}^{N} c_{k \ell} \Lambda_{\ell}\left|\Lambda_{\ell}\right\rangle+\delta \boldsymbol{U}_{0}\left(\kappa_{2}\right)\left|\Lambda_{k}\right\rangle=\Lambda_{k} \sum_{\ell=0}^{N} c_{k \ell}\left|\Lambda_{\ell}\right\rangle \\
+\delta \Lambda_{k}\left|\Lambda_{k}\right\rangle .
\end{gathered}
$$

Now, we can apply the orthonormality condition $\left\langle\Lambda_{m} \mid \Lambda_{\ell}\right\rangle=\delta_{m \ell}$ from Eq. (21), to Eq. (26) to get

$$
c_{k m} \Lambda_{m}+\left\langle\Lambda_{m}\left|\delta \boldsymbol{U}_{0}\left(\kappa_{2}\right)\right| \Lambda_{k}\right\rangle=c_{k m} \Lambda_{k}+\delta \Lambda_{k} \delta_{m k} .
$$

Therefore, the shift in the eigenvalue is found when $m=k$,

$$
\delta \Lambda_{k}=\left\langle\Lambda_{k}\left|\delta \boldsymbol{U}_{0}\left(\kappa_{2}\right)\right| \Lambda_{k}\right\rangle
$$

and the change in eigenvectors is found when $m \neq k$,

$$
c_{k m}=\frac{\left\langle\Lambda_{m}\left|\delta \boldsymbol{U}_{0}\left(\kappa_{2}\right)\right| \Lambda_{k}\right\rangle}{\Lambda_{k}-\Lambda_{m}} .
$$

Thus using Eq. (25), we have

$$
\left|\delta \Lambda_{k}\right\rangle=\sum_{\ell \neq k}^{N} \frac{\left\langle\Lambda_{\ell}\left|\delta \boldsymbol{U}_{0}\left(\kappa_{2}\right)\right| \Lambda_{k}\right\rangle}{\Lambda_{k}-\Lambda_{\ell}}\left|\Lambda_{\ell}\right\rangle
$$

where we are allowed to choose $c_{k k}=0$ because $\left\langle\Lambda_{k}^{\prime} \mid \Lambda_{k}^{\prime}\right\rangle=\left\langle\Lambda_{k} \mid \Lambda_{k}\right\rangle=1$ to first order.

\section{Eigenfrequency of $\pi$ mode for $\kappa_{2} \ll \kappa_{1}$}

The correction to the eigenvalue $\Lambda_{k}$ comes from Eq. (28) and is

$$
\begin{aligned}
\delta \Lambda_{k} & =\left\langle\Lambda_{k}\left|\delta \boldsymbol{U}_{0}\left(\kappa_{2}\right)\right| \Lambda_{k}\right\rangle \\
& =\left(\boldsymbol{J}^{-1}\right)_{k} \delta \boldsymbol{U}_{0}\left(\kappa_{2}\right) \boldsymbol{J}_{k} .
\end{aligned}
$$

For $\pi$ mode, we have $k=N$ and when we multiply out Eq. (31), we find that

$$
\delta \Lambda_{N}=-\frac{2 \kappa_{2}(N-1)}{N} .
$$

We recall that the eigenfrequency $\Omega_{k}$ is related to $\Lambda_{k}+\delta \Lambda_{k}$ by

$$
\Omega_{k}=\frac{\omega_{c}}{\sqrt{\Lambda_{k}+\delta \Lambda_{k}}}
$$

and so, for $\pi$ mode, we have $k=N$ and thus

$$
\Lambda_{N}=1+2 \kappa_{1} .
$$

Therefore, the corrected eigenfrequency that takes into account both $\kappa_{1}$ and $\kappa_{2}$ is

$$
\Omega_{N}=\frac{\omega_{c}}{\sqrt{\left(1+2 \kappa_{1}\right)-2 \kappa_{2}(N-1) / N}} .
$$

\section{Eigenvector correction of $\pi$-mode eigenvector for $\kappa_{2} \ll \kappa_{1}$}

The correction to the eigenvector $\left|\Lambda_{k}\right\rangle$ comes from Eq. (30) and is

$$
\begin{aligned}
\left|\delta \Lambda_{k}\right\rangle & =\sum_{\ell \neq k}^{N} \frac{\left\langle\Lambda_{\ell}\left|\delta \boldsymbol{U}_{0}\left(\kappa_{2}\right)\right| \Lambda_{k}\right\rangle}{\Lambda_{k}-\Lambda_{\ell}}\left|\Lambda_{\ell}\right\rangle \\
& =\sum_{\ell \neq k}^{N} \frac{\left(\boldsymbol{J}^{-1}\right)_{\ell} \delta \boldsymbol{U}_{0}\left(\kappa_{2}\right) \boldsymbol{J}_{k}}{\Lambda_{k}-\Lambda_{\ell}} \boldsymbol{J}_{\ell} .
\end{aligned}
$$

We notice that

$$
\left(\boldsymbol{J}^{-1}\right)_{\ell} \delta \boldsymbol{U}_{0}\left(\kappa_{2}\right) \boldsymbol{J}_{N} \propto \kappa_{2}
$$

because $\boldsymbol{J}_{k}$ is independent of both $\kappa_{1}$ and $\kappa_{2}$, and from Eq. (16), we have

$$
\Lambda_{N}-\Lambda_{\ell}=\left\{\begin{array}{rr}
2 \kappa_{1}\left(1+\cos \frac{\ell \pi}{N}\right) & \text { if } \ell \neq 0 \\
4 \kappa_{1} & \text { if } \ell=0 .
\end{array}\right.
$$

Therefore,

$$
\frac{\left(\boldsymbol{J}^{-1}\right)_{\ell} \delta \boldsymbol{U}_{0}\left(\kappa_{2}\right) \boldsymbol{J}_{N}}{\Lambda_{N}-\Lambda_{\ell}} \propto \frac{\kappa_{2}}{\kappa_{1}} \Rightarrow\left|\delta \Lambda_{N}\right\rangle \propto \frac{\kappa_{2}}{\kappa_{1}} \equiv \mu .
$$

Hence, we have demonstrated that, as long as $\mu$ remains constant, the correction $\left|\delta \Lambda_{N}\right\rangle$ remains constant, and this is the reason why the curvature that will be shown later in Fig. 9 remains the same when $\kappa_{1}$ is changed while keeping $\mu$ constant. 




FIG. 7 (color online). This is a view of the FNAL RFQ that shows the tuning plates at arbitrary heights $h_{n}$.

\section{The effect of changing the height of any tuning plate}

The effect on the eigenfrequencies and eigenvectors for arbitrary tuning plate positions will be studied in this section. Let us denote the height of the tuning plate in cell $n$ to be $h_{n}$ and when all the tuning plates are at their lowest position, i.e. $h_{n}=0$, the inductance $L_{n}=L$ for $n=0, \ldots . ., N$, and the capacitance for cells $n=1, \ldots ., N-1$ is $C_{n}=C$ and $C_{0}=C_{N}=2 C$. Now, unlike in previous sections, we must allow for arbitrary heights $h_{n}$ (see Fig. 7). This means that the inductance in cell $n$ becomes

$$
L_{n} \rightarrow L+\delta L_{n},
$$

where $\delta L_{n}$ is the change in inductance in cell $n$ from $L$.

From Eq. (5), we have two types of terms to consider. The first type comes from the diagonal of $\boldsymbol{U}_{1}$ which is

$$
L_{n} C_{n}=\left(L+\delta L_{n}\right) C \Rightarrow L_{n} C_{n}=L C\left(1+\frac{\delta L_{n}}{L}\right) \text {. }
$$

We can write the above in terms of the base cell frequency $\omega_{c}^{2}=1 / L C$ and the new base frequency $\omega_{n}{ }^{\prime}$ :

$$
\begin{aligned}
\omega_{n}{ }^{\prime} & =\frac{\omega_{c}}{\left(1+\frac{\delta L_{n}}{L}\right)^{1 / 2}} \approx \omega_{c}\left(1-\frac{1}{2} \frac{\delta L_{n}}{L}\right) \quad \text { for } \delta L_{n} \ll L \\
\Rightarrow \frac{\delta L_{n}}{L} & =-\frac{2\left(\omega_{n}{ }^{\prime}-\omega_{c}\right)}{\omega_{c}} \equiv-\delta_{n} .
\end{aligned}
$$

The advantage of using frequency changes rather than inductance changes when populating the entries of $\boldsymbol{U}_{1}$ is that the form of $\boldsymbol{U}_{1}$ remains unchanged when the $C_{n}$ 's are perturbed.

Therefore, $L_{n} C_{n}$ in terms of the base cell frequency $\omega_{c}$ and its shift $\delta_{n}$ is

$$
L_{n} C_{n}=\frac{1}{\omega_{c}^{2}}\left(1-\delta_{n}\right) .
$$

The second type comes from the off-diagonal of $\boldsymbol{U}_{1}$ which is

$$
\begin{aligned}
\sqrt{L_{n} L_{m}} C & =\sqrt{\left(L+\delta L_{n}\right)\left(L+\delta L_{m}\right)} C \\
& \approx L C \sqrt{\left(1+\frac{\delta L_{n}}{L}\right)\left(1+\frac{\delta L_{m}}{L}\right)} \\
& =\frac{1}{\omega_{c}^{2}}\left[1-\frac{1}{2}\left(\delta_{n}+\delta_{m}\right)\right] \\
& \equiv \frac{1}{\omega_{c}^{2}}\left(1-\frac{1}{2} \Delta_{n m}\right),
\end{aligned}
$$

where we have neglected the $\left(\delta L_{n}\right)\left(\delta L_{m}\right) / L^{2}$ term.

When we substitute Eqs. (43) and (44) into Eq. (5), we have

$$
\boldsymbol{U}_{1}=\lambda_{c}^{2}\left[\boldsymbol{U}_{0}\left(\kappa_{1}, 0\right)+\delta \boldsymbol{U}_{0}\left(\kappa_{2}\right)+\delta \boldsymbol{W}_{0}\left(\kappa_{1}, \kappa_{2}\right)\right],
$$

where 
Now, we can apply exactly the same methods discussed in Sec. IIB using perturbation theory to calculate the effect of changes in $L_{n}$. The correction to the eigenvalues $\Lambda_{k}$ comes from Eq. (28) and is

$$
\delta \Lambda_{k}=\left(\boldsymbol{J}^{-1}\right)_{k}\left[\delta \boldsymbol{U}_{0}\left(\kappa_{2}\right)+\delta \boldsymbol{W}_{0}\left(\kappa_{1}, \kappa_{2}\right)\right] \boldsymbol{J}_{k}
$$

and the correction to the eigenvectors comes from Eq. (30):

$$
\left|\delta \Lambda_{k}\right\rangle=\sum_{\ell \neq k}^{N} \frac{\left(\boldsymbol{J}^{-1}\right)_{\ell}\left[\delta \boldsymbol{U}_{0}\left(\kappa_{2}\right)+\delta \boldsymbol{W}_{0}\left(\kappa_{1}, \kappa_{2}\right)\right] \boldsymbol{J}_{k}}{\Lambda_{k}-\Lambda_{\ell}} \boldsymbol{J}_{\ell} .
$$

We will compare these perturbative solutions to the numerical solution of the same eigensystem in Sec. IV.

If we continue further, and after quite a bit of expansion and simplification of Eq. (48), we find that when only the $n$th plate is moved, the correction when expanded to first order in $\delta_{n}$ is

$$
\sum_{\ell \neq k}^{N} \frac{\left(\boldsymbol{J}^{-1}\right)_{\ell} \delta \boldsymbol{W}_{0}\left(\kappa_{1}, \kappa_{2}\right) \boldsymbol{J}_{k}}{\Lambda_{k}-\Lambda_{\ell}} \boldsymbol{J}_{\ell}=\delta_{n} \sum_{\ell \neq k}^{N} w_{\ell}\left(\kappa_{1}, \kappa_{2}, n\right) \boldsymbol{J}_{\ell},
$$

where $w_{\ell}$ are functions independent of $\delta_{n}$. The above relationship turns out to be important for understanding and explaining the idiosyncrasies of the superposition method that has been historically used to predict the field profile. For example, it can be shown using Eq. (49) that the $H$ function mentioned in Refs. [3] and [12] is, in fact, $\delta_{n}$.

Furthermore, by linearizing the problem, we have made methods such as Gauss-Newton available to us for the optimization of either $L_{n}$ or $C_{n}$ (in the form of $\delta_{n}$ ) to fit to simulated or measured eigenvectors.

\section{USING THE MODEL}

In order to use our model, we must calculate $L_{n}, C_{n}, \kappa_{1}$, $\kappa_{2}$, and $\delta L_{n} / L$ using the results from MWS and then refine these values by using the results from measurements. The FNAL RFQ will serve as the structure for this exercise [13]. Before we begin, we need to discuss the duality of $L_{n}$ and $C_{n}$. Duality helps by allowing us to keep either $L_{n}$ or $C_{n}$ constant and then varying the remaining variable for fitting purposes.

\section{A. Duality of $L_{n}$ and $C_{n}$}

We notice that the natural frequency of each isolated tuning cell is $\omega_{n}^{\prime}=1 / \sqrt{L_{n} C_{n}}$ for $n=1, \ldots . ., N-1, \omega_{0}^{\prime}=$ $1 / \sqrt{L_{0} C_{0} / 2}$ and $\omega_{N}^{\prime}=1 / \sqrt{L_{N} C_{N} / 2}$, i.e.

$$
\omega_{n}^{\prime 2} \propto \frac{1}{L_{n} C_{n}} \quad \text { for } k=0, \ldots, N .
$$

We can think of $L_{n}$ and $C_{n}$ as mathematical duals because any variation of the tuning cell from the ideal can either be assigned as an error to either $L_{n}$ or $C_{n}$.

\section{B. Fits to MWS data}

In the first pass, we will assume that $C_{n}=C$ for $n=1, \ldots ., N-1, C_{0}=C_{N}=2 C$ to allow us to calculate $\kappa_{1}, \kappa_{2}$, and $\delta L_{n} / L$ by fitting the model to the MWS results. In the second pass, where we have to match our model to the measured field flatness, we will vary the capacitance of each cell to get a good fit. Note, the MWS model of the RFQ used in this section does not contain any modulations and so the field flatness profile will be symmetric about cell 6 when all the tuning plates are at the same height.

\section{Value of $C$}

We have to set some value to $C$ in order to get the ball rolling. There are several ways to calculate the capacitance of the system with simplified electrodes in the form of circular rods. The solutions to these models are typically derived with the theory of cables or image charges, like the examples given in [14]. Instead of using these simplified models, we have simulated the capacitance of the electrodes using CST EM STUDIO ${ }^{\circledR}$ (EMS). EMS exports a capacitance matrix of the system which includes the separated lumped capacitance between each defined potential as shown in Fig. 8.

According to the quad star model, also shown in Fig. 8, we can describe the quadrupole as a capacitance $C_{A}$, consisting of the four parallel capacitors $c_{i j}$ between the electrodes,

$$
C_{A}=c_{12}+c_{23}+c_{34}+c_{41},
$$

and are in series with two capacitors $C_{B}$ and $C_{C}$ :

$$
\begin{aligned}
& C_{B}=c_{11}+c_{33}, \\
& C_{C}=c_{22}+c_{44} .
\end{aligned}
$$

$C_{B}$ and $C_{C}$ represent the capacitance between two pairs of electrodes $(V 1, V 3),(V 2, V 4)$ and the boundary.

These capacitors $C_{A}, C_{B}$, and $C_{C}$ form a $\pi$ network as one can find for example in [14] and with $V 1=V 3=$ $-V 2=-V 4$ it leads to a capacitance of the electrodes $C_{T}$ described by

$$
C_{T}=C_{A}+\frac{C_{B} C_{C}}{C_{B}+C_{C}} .
$$

When we substitute the values, given in the capacitance matrix of EMS, into Eqs. (51)-(53), we find that $C_{T}=$ $124.6 \mathrm{pF}$ and thus the lumped circuit capacitance $C=$ $C_{T} / 15=8.3 \mathrm{pF}$ because the total capacitance of the RFQ lumped circuit model is $C_{T}=11 C+2 \times 2 C=15 C$. 

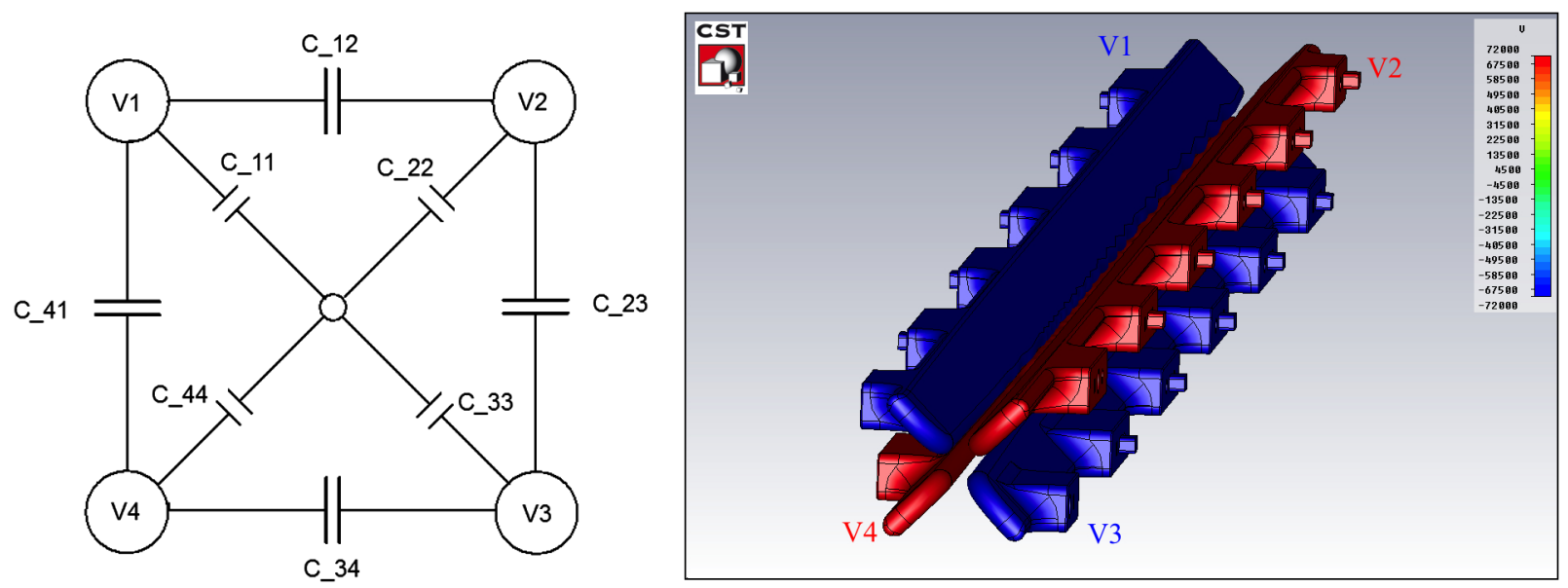

FIG. 8 (color online). For the purpose of calculating $C$ with EMS, we have set one pair of electrodes to $72 \mathrm{kV}$ (red) and another pair to $-72 \mathrm{kV}$ (blue). From here, we can extract the lumped capacitance matrix to calculate $C_{T}$ using the star quad model.

\section{Extracting $\kappa_{1}$ and $\kappa_{2}$}

We can extract $\kappa_{1}$ and $\kappa_{2}=\mu \kappa_{1}$ by using the MWS results for the case when all the tuning plates are at zero. In addition, we will also keep the resonant frequency fixed at $154.733 \mathrm{MHz}$ found by MWS in this configuration. The goal is to perform a least square fit to minimize the difference between the $\pi$-mode eigenvector $\boldsymbol{J}_{12}$ and the MWS eigenvector by varying $\kappa_{1}$ and $\mu$ with the given constraint.

The results of the fit are $\kappa_{1}=0.0936$ and $\mu=0.03351$ and are shown in Fig. 9(a). We also illustrate in Fig. 9(b) that when $\kappa_{1} \rightarrow\left(10 \times \kappa_{1}=0.936\right)$ but with $\mu$ kept constant, the fit remains good, but the resonant frequency has changed to $163.793 \mathrm{MHz}$. This result supports the point that was made earlier in Sec. IIB5 and Eq. (39) that to first order, $\kappa_{1}$ determines the resonant frequency while $\mu$ determines the curvature of the field.

As a consequence of the choice of $C$ and the fit, $L=$ $0.108 \mu \mathrm{H}$ when the above values are substituted into
Eq. (35). Table I summarizes all the results of the fits to the MWS data.

\section{Constructing $\delta L$ as a function of $h$}

The resonant frequency change of the RFQ when all its tuning plates are raised to the same height, shown in Fig. 10, indicates to us that $\delta L$ must be at least a quadratic function of the tuning plate height $h$. Therefore, let us define two free parameters $a$ and $b$ in the following equation:

$$
\delta L(h)=L\left(a h^{2}+b h\right)
$$

that will be found by fitting to the MWS data described below. Reminder-we have assumed that $\delta L(h)$ is the same for all tuning cells.

We choose to find $a$ and $b$ by applying a least squares fit that minimizes the difference between the model relative voltage, $\left|V_{3}\left(h_{3}\right) / V_{3}(0)\right|$, and the same ratio found by
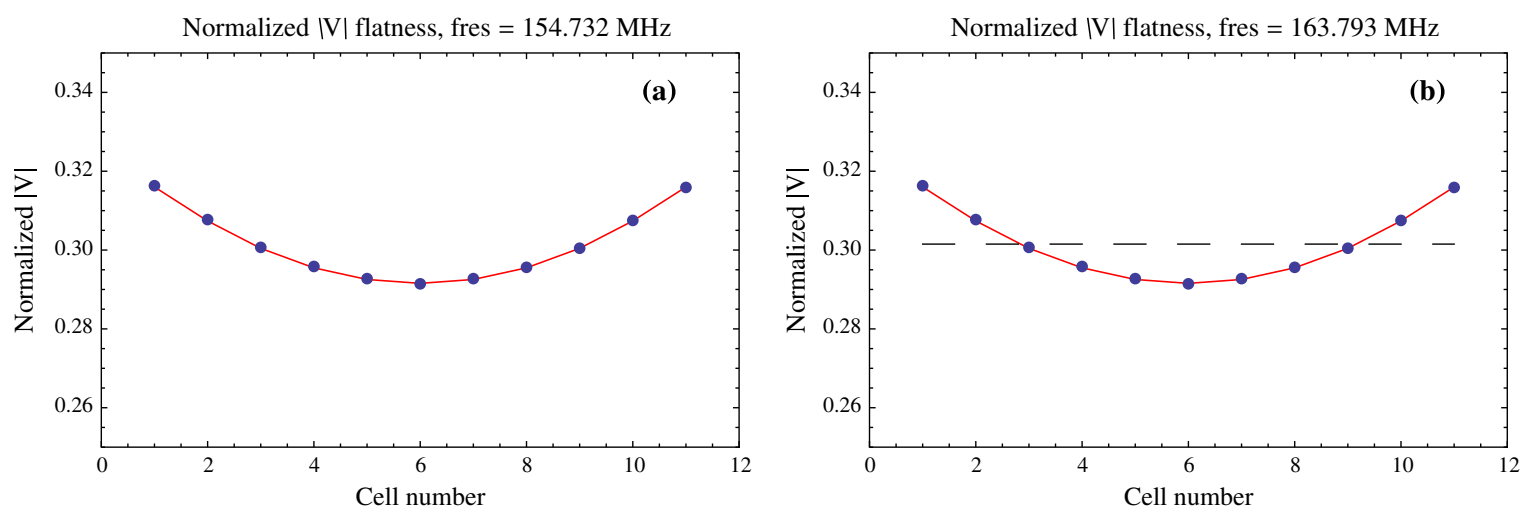

FIG. 9 (color online). (a) The red curve is the result of fitting the model to the MWS field flatness profile (blue filled dots) while keeping the resonant frequency fixed. (b) $\kappa_{1}$ is increased by $10 \times$ while $\mu$ has the same value that is used to create (a). The curve from (a) is still good but the resonant frequency has shifted. The dashed line represents the case when $\mu=0$ and shows that there is no curvature in this case. 


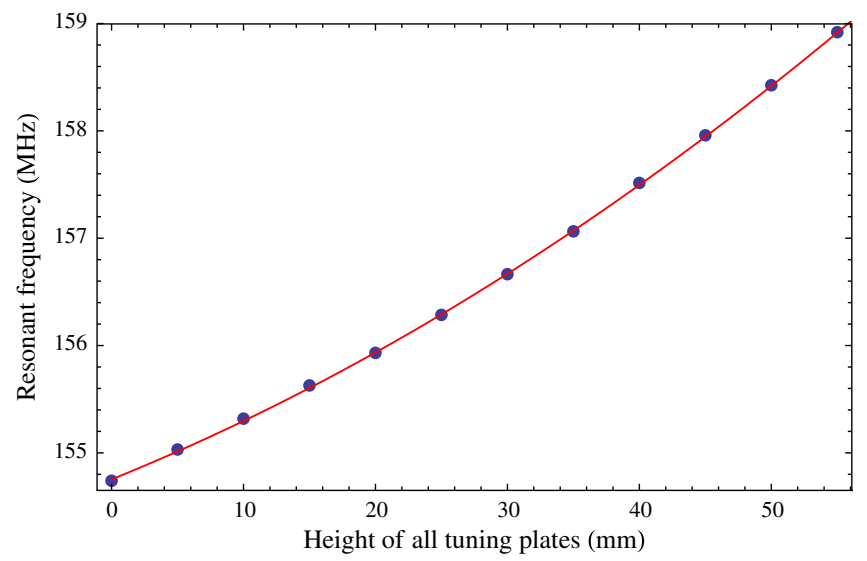

FIG. 10 (color online). The resonant frequency when all the tuning plates are raised to the same height calculated by MWS. When all the tuning plates are at $0 \mathrm{~mm}$, the resonant frequency is 154.7 and $158.4 \mathrm{MHz}$ at $50 \mathrm{~mm}$. The red curve is a quadratic fit to the data.

MWS. We define $V_{3}\left(h_{3}\right)$ to be the voltage in cell 3 when the tuning plate is at $h_{3}$ while all the other tuning plates are at zero height. The fit gives

$$
a=-1.00 \times 10^{-6} \mathrm{~mm}^{-2} \quad b=-0.38 \times 10^{-4} \mathrm{~mm}^{-1}
$$

and Fig. 11(a) shows the result of the least squares fit plotted with the MWS data. The range of $\delta L(h) / L$ is much smaller than expected and it has the consequence that the resonant frequency is not predicted correctly as a function of tuning plate height. This is easily shown when we use Eq. (42) and the values of the resonant frequencies from Fig. 10,

$$
\begin{aligned}
\frac{\delta L}{L} & =-\frac{2\left(f_{\text {res }}(50)-f_{\text {res }}(0)\right)}{f_{\text {res }}(0)}=-\frac{2(158.4-154.7)}{154.7} \\
& =-0.048,
\end{aligned}
$$

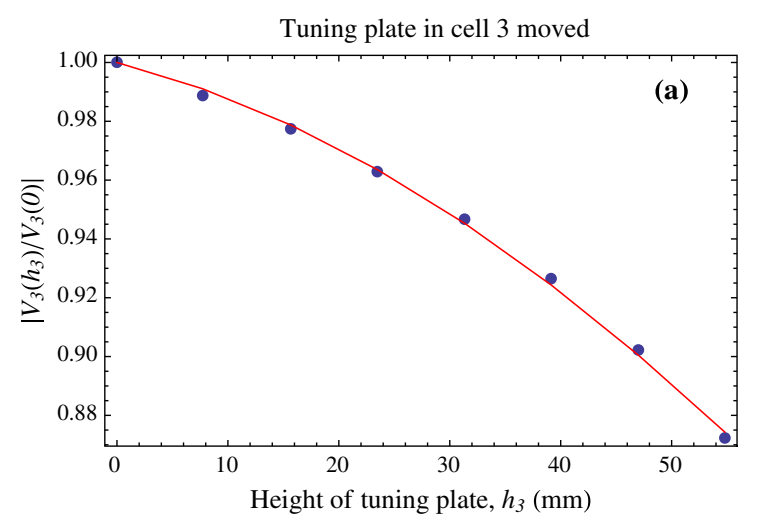

where $f_{\text {res }}(h)$ is the resonant frequency calculated by MWS when all the plates are at $h \mathrm{~mm}$. However, when we substitute the $a$ and $b$ values in Eq. (55) into Eq. (54), we get $\frac{\delta L}{L}=-0.0044$ which is $10 \times$ smaller than what we expect. This is a weakness of the model that will need to be addressed in the future.

\section{COMPARISON OF THE MODEL TO THE MWS RESULTS}

As a comparison, we can apply our model with the parameters found in the previous sections and summarized in Table I to other MWS results. Figure 12 shows a selection of MWS data that are plotted against the predicted curves of our model by numerically solving the eigensystem given by Eq. (5) and by perturbation theory described in Sec. IIB3. We can see that the predicted curves of both numerical solution and the perturbation solution can match the MWS data quite well. However, clearly, both methods do not always match the MWS data exactly. Sometimes, one method seems to be better than the other. The discrepancies are highlighted in the caption of that figure. We can certainly make the curves fit better to the MWS data by varying the capacitance of each cell but this effort is better expended on fitting the curves to measured data. This will be the topic of the next section.

\section{FITTING THE MODEL TO MEASURED RESULTS}

The list of capacitance corrections that are needed for the model to fit to the measured relative voltage [15] is $\Delta \boldsymbol{C}=\left\{\Delta C_{n} \mid n=0, \ldots, 12\right\}$. We will only consider the cases where tuning plates 3 and 9 have been set to $20 \mathrm{~mm}$ because we want to determine whether our fit to a subset of the data set is sufficient to predict the voltage behavior when applied to the remaining data set. Figure 13 shows the $\Delta \boldsymbol{C}$ required for fitting to this subset. Note, we have left out the voltage effects of tuning plates 0 and 12 because we did not measure their effects.

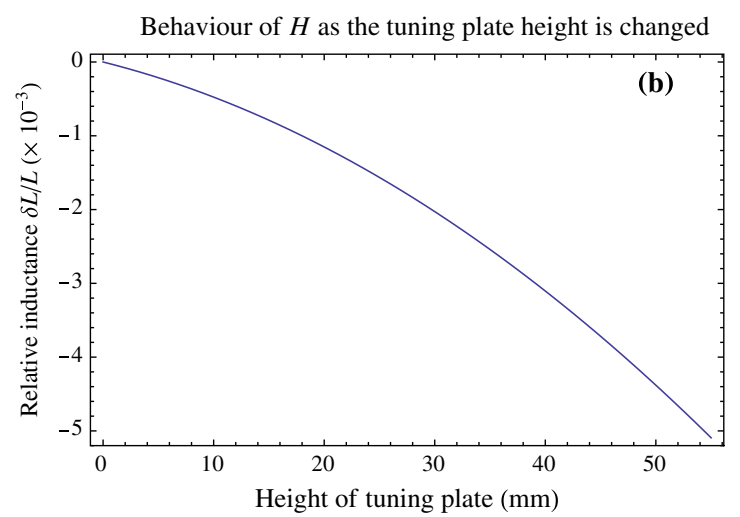

FIG. 11 (color online). (a) The red curve is the result of fitting the model to the MWS data (blue filled dots) when tuning plate 3 is moved. Part (b) is the corresponding $\delta L / L$ found from the fit. See text. 



FIG. 12 (color online). All the curves have been calculated using the parameters shown in Table I and the filled dots, filled squares, etc. come from MWS. All the continuous curves come from numerically solving Eq. (4) and the dashed curves come from perturbation theory solutions described in Sec. IIB3. (a) The relative field flatness when tuning plate 3 is at $30 \mathrm{~mm}$ while the rest are at $0 \mathrm{~mm}$. (b) The relative voltage in tuning cells 1 and 6 when their tuning plates are moved. (c) The relative field flatness of all the cells when the tuning plate in cell 3 is set to 31 and $55 \mathrm{~mm}$. The predicted curves are good for tuning cells 4 to 11 . Interestingly, the perturbative solution matches the MWS data better than the numerical eigensystems solution. (d) The relative voltage response of tuning cells 1,4 , 7, and 11 when tuning plate 3 is moved. The predicted curves using the numerical eigensystems method match the MWS data very well except for cell 1 , while the perturbative solution does not do as well.

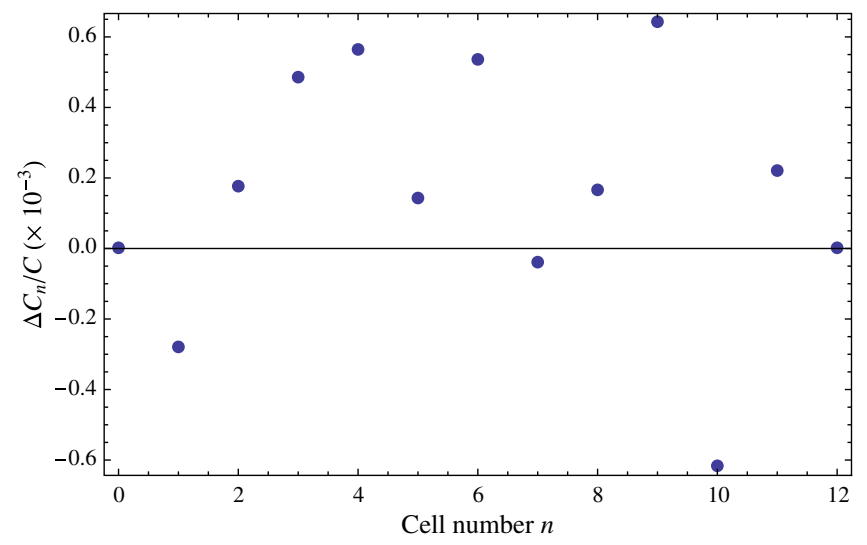

FIG. 13 (color online). The relative change $\Delta C_{n} / C$ for $n=$ $1, \ldots . ., 11$ that is needed to minimize the difference between the predicted relative voltage and the measurements when tuning plates 3 and 9 are individually set to $20 \mathrm{~mm}$. The results of the fit are shown in Fig. 14. Note, we did not use the effect of tuning plates 0 and 12 in the fits.
The results presented in Fig. 14 illustrate that the relative changes to the capacitance are asymmetric about tuning cell 6 and are very small when compared to $C$. We believe that both the asymmetry and $\Delta C_{n} \ll C$ indicate the influence of the growing modulation [2] and to second order, the manufacturing and mounting errors of the rods.

The model predictions using $\Delta \boldsymbol{C}$ are shown in Fig. 14. When we compare the predicted results to the measurements, we see that the model and measurements match extremely well. These results set us up for comparing the model with these parameters to the measurements for arbitrarily set tuning plate heights. This will be discussed in the next section.

\section{COMPARISON OF THE MODEL PREDICTED FIELD FLATNESS TO MEASUREMENT}

In this section, we will compare our model prediction to measurements by the simple and direct way of numerically solving the eigensystem equation derived in Sec. IIA after filling in $\boldsymbol{U}_{1}$ with the values from Table I, $\delta L_{n} / L$ and $\Delta \boldsymbol{C}$ found in the previous sections. We will not delve into detail 

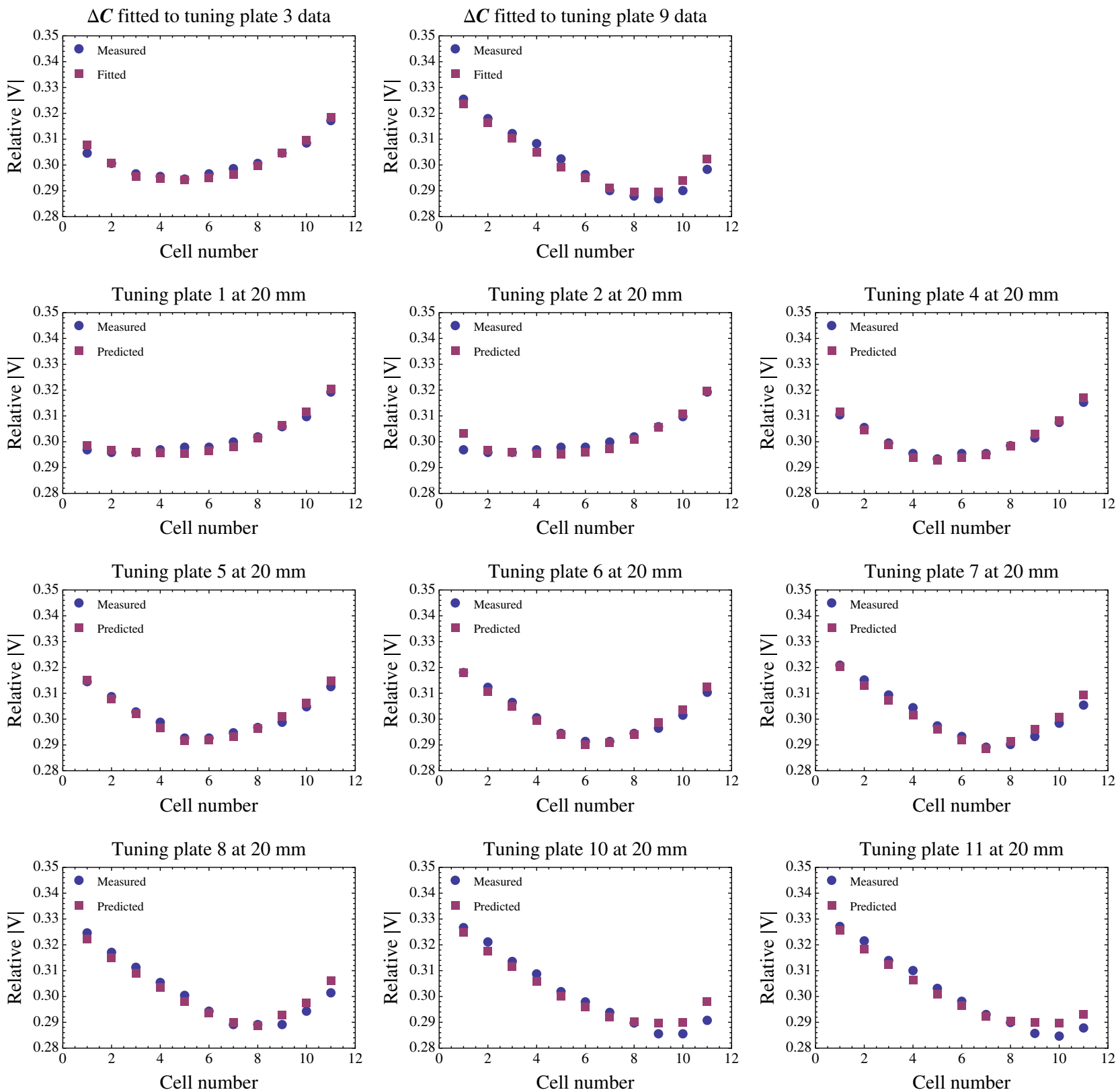

FIG. 14 (color online). We fit $\Delta \boldsymbol{C}$ to the measured relative field profile for the cases when the tuning plates in cells 3 and 9 were set to $20 \mathrm{~mm}$. We then used the $\Delta \boldsymbol{C}$ that we found (see Fig. 13) and applied it to the model. The results are plotted here for the other cells when each plate was raised to $20 \mathrm{~mm}$. We can see that the predicted relative voltage and the measured relative voltage match extremely well.

about how to accomplish the extraction of the eigenvectors of $\boldsymbol{U}_{1}$ because many canned packages are able to do this easily. The model predictions compared to three measurements with arbitrary tuning plate heights are shown in Fig. 15.

In order to test the hypothesis that results of the model can predict the measurements, we will use the reduced $\chi^{2}$ goodness of fit test [16]. The definition of the reduced $\chi^{2}$ is

$$
\chi^{2} / \nu=\frac{1}{\nu} \sum_{n=1}^{11} \frac{\left(\tilde{V}_{\text {measured }}(n)-\tilde{V}_{\text {predicted }}(n)\right)^{2}}{\sigma_{n}^{2}},
$$

where $\tilde{V}_{\text {measured }}(n)$ and $\tilde{V}_{\text {predicted }}(n)$ are the measured relative voltage and the predicted relative voltage in cell $n$, respectively, $\sigma_{n}^{2}$ is the variance of the measurement in cell $n$, and $\nu$ is the number of degrees of freedom.

In all the example setups, $\nu=$ number of tuning plates $=$ 11 because we are not fitting or deriving any statistical quantities from the measurements. When we choose the significance value $\alpha=0.05$, the critical value $\chi^{2} / \nu_{\text {crit }}=1.78$. We will use $\chi^{2} / \nu_{\text {crit }}$ to determine whether the models acceptably describe the measurements. For calculating $\chi^{2} / \nu$, we will assume that the measurements have a standard error of $1 \%$. 

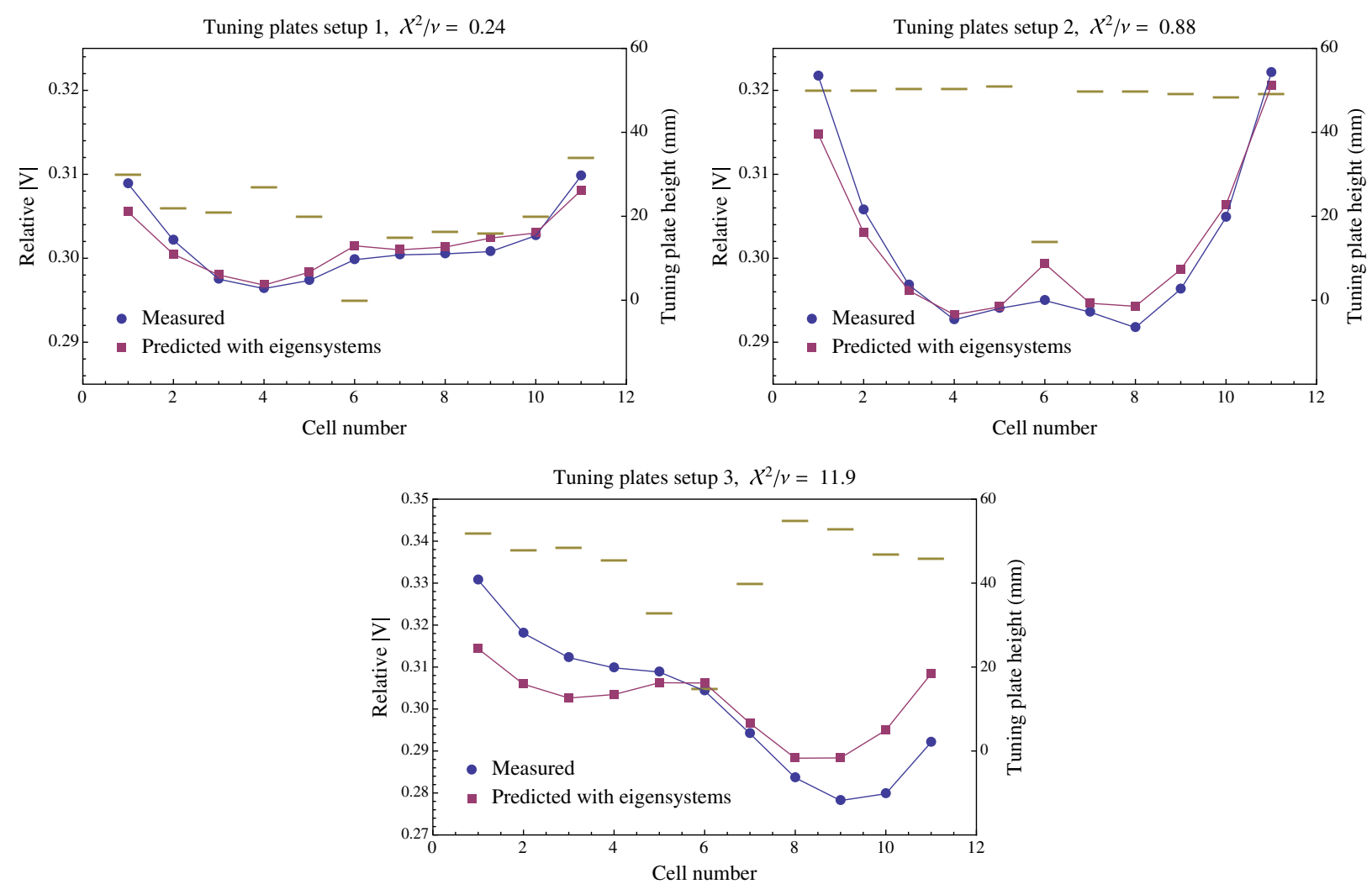

FIG. 15 (color online). The comparison between our model predictions and measurements for three different tuning plates setups are shown here. The - in these graphs shows the tuning plate heights. Because $\chi^{2} / \nu<\chi^{2} / \nu_{\text {crit }}=1.78$ for setups 1 and 2 , the model matches the measurements. But $\chi^{2} / \nu \gg 1$ in setup 3 and the model prediction is unacceptable although the shape of the profile does mimic the measurement.

For setup 1, where most of the tuning plate heights are close to $20 \mathrm{~mm}, \chi^{2} / \nu=0.24$ of our model is $<\chi^{2} / \nu_{\text {crit }}$, and so we do not reject our models at the 5\% significance level. We can see by eye that the model predictions do indeed match the measurements very well.

For setup 2, we have raised most of the tuning plates to about $50 \mathrm{~mm}$. Again, the model $\chi^{2} / \nu=0.88<\chi^{2} / \nu_{\text {crit }}$ and so we do not reject this model at the $5 \%$ significance level.

For setup 3, we see that the model does not match the measurements very well at the 5\% significance level because $\chi^{2} / \nu=11.9>\chi^{2} / \nu_{\text {crit }}$. However, the shape of the prediction correlates with the measurements. This indicates that $\Delta \boldsymbol{C}$ needs further refinement.

Therefore, from these three setups, we can see that our model is usable for predicting the shape of the field profile. For two of our three setups, the model predictions match the measurements. In fact, in practice, we can imagine after each setup and measurement, the value of the capacitance used for each cell can be refined to make the predicted profile match the measured profile. Then, with the new set of capacitances, we can use it to predict the next profile for a new set of tuning plate heights. These iterations that consist of the sequence of prediction and correction form the basis of a tuning algorithm.

\section{CONCLUSION}

We have shown how the application of perturbation theory to the lumped circuit model can be used to understand the physics of field flatness tuning. Because the numerical solution of eigensystems is very fast, the model also provides the opportunity for real-time calculation of the field flatness profile. When we couple the real-time calculation with an iterative process of prediction and correction, the model can be quickly refined to give accurate results. This procedure makes it possible for the RFQ expert to not only use the model to tune the field flatness to some tolerance but also to shape it. This would be desirable because there are CST PARTICle STUdio ${ }^{\circledR}$ simulations as well as PARMTEQ calculations that reveal the capture and transmission efficiency of the RFQ is impacted by the shape of the field profile [12,17].

Of course, there are also improvements that need to be made to the model. For example, the present model does not predict the resonant frequency correctly. Currently, we are developing a LABVIEW program that implements this model and will be used to tune newly built fourrod RFQs. 


\section{ACKNOWLEDGMENTS}

We would like to thank R. Madrak for proofreading our manuscript. This work is supported by Fermi Research Alliance, LLC under Contract No. DE-AC02-07CH11359 with the U.S. Department of Energy and the Bundesministerium für Bildung und Forschung (BMBF).

\section{APPENDIX A: CONSTRUCTING THE EIGENBRA $\left\langle J_{k}\right|$}

Let us write down the eigenvectors $\boldsymbol{J}_{k}$ of $\boldsymbol{U}_{0}\left(\kappa_{1}, 0\right)$ as eigenkets, i.e.

$$
\boldsymbol{J}_{k}=\left|\Lambda_{k}\right\rangle
$$

Unfortunately, $\left\{\left|\Lambda_{k}\right\rangle \mid k=0, \ldots . ., N\right\}$ do not form an orthonormal set because $\boldsymbol{U}_{0}\left(\kappa_{1}, 0\right)$ is not symmetric. However, we can always construct a set of eigenbras $\left\{\left\langle\Lambda_{k}|| k=\right.\right.$ $0, \ldots ., N\}$ that are orthogonal to $\left\{\left|\Lambda_{k}\right\rangle \mid k=0, \ldots . ., N\right\}$ if its eigenvalues are distinct. In this paper, it is, indeed, the case from Eq. (16).

We start with the similarity transformation that diagonalizes $\boldsymbol{U}_{0}\left(\kappa_{1}, 0\right)$ from Eq. (15):

$$
\underline{\boldsymbol{J}}^{-1} \boldsymbol{U}_{0}\left(\kappa_{1}, 0\right) \boldsymbol{J}=\Lambda \Rightarrow \boldsymbol{U}_{0}\left(\kappa_{1}, 0\right) \boldsymbol{J}=\boldsymbol{J} \Lambda \quad \text { and } \quad \underline{J}^{-1} \boldsymbol{U}_{0}\left(\kappa_{1}, 0\right)=\Lambda \boldsymbol{J}^{-1} \text {. }
$$

Therefore, from the last line of Eq. (A2), we can see that the $(k+1)$ th column of $\boldsymbol{J}$ is the right eigenvector of $\boldsymbol{U}_{0}\left(\kappa_{1}, 0\right)$ associated with eigenvalue $\Lambda_{k}$ and the $(k+1)$ th row vector of $\boldsymbol{J}^{-1}$ is the left eigenvector of $\boldsymbol{U}_{0}\left(\kappa_{1}, 0\right)$ associated with the same eigenvalue $\Lambda_{k}$.

Hence, if we define $\left(\boldsymbol{J}^{-1}\right)_{k}$ to be the $(k+1)$ th row of $\boldsymbol{J}^{-1}$, we can write

$$
\left(\boldsymbol{J}^{-1}\right)_{k}=\left\langle\Lambda_{k}\right|
$$

and it is clear from $\boldsymbol{J}^{-1} \boldsymbol{J}=\boldsymbol{I}$ so that

$$
\left\langle\Lambda_{k} \mid \Lambda_{\ell}\right\rangle=\left(\boldsymbol{J}^{-1}\right)_{k} \boldsymbol{J}_{\ell}=\delta_{k \ell},
$$

where $\delta_{k \ell}$ is the Kronecker $\delta$.

Thus, we have found a set of eigenbras $\left\{\left\langle\Lambda_{k} \| k=\right.\right.$ $0, \ldots ., N\}$ that are orthonormal to the eigenkets $\left\{\left|\Lambda_{k}\right\rangle \mid k=0, \ldots . ., N\right\}$.

\section{APPENDIX B: $J$ and $J^{-1}$ IN MATRIX FORM}

$$
\begin{aligned}
& \boldsymbol{J}=\left(\begin{array}{ccccccc}
A_{0} & A_{1} & A_{2} & \cdots & A_{k} & \cdots & A_{N} \\
A_{0} & A_{1} \cos \frac{\pi}{N} & A_{2} \cos \frac{2 \pi}{N} & \cdots & A_{k} \cos \frac{k \pi}{N} & \cdots & -A_{N} \\
A_{0} & A_{1} \cos \frac{2 \times \pi}{N} & A_{2} \cos \frac{2 \times 2 \pi}{N} & \cdots & A_{k} \cos \frac{2 \times k \pi}{N} & \cdots & A_{N} \\
\vdots & \vdots & \vdots & & \vdots & & \vdots \\
A_{0} & A_{1} \cos \frac{(N-2) \pi}{N} & A_{2} \cos \frac{(N-2) 2 \pi}{N} & \cdots & A_{k} \cos \frac{(N-2) k \pi}{N} & \cdots & (-1)^{N-2} A_{N} \\
A_{0} & A_{1} \cos \frac{(N-1) \pi}{N} & A_{2} \cos \frac{(N-1) 2 \pi}{N} & \cdots & A_{k} \cos \frac{(N-1) k \pi}{N} & \cdots & (-1)^{N-1} A_{N} \\
A_{0} & -A_{1} & A_{2} & \cdots & (-1)^{k} A_{k} & \cdots & (-1)^{N} A_{N}
\end{array}\right) \\
& \boldsymbol{J}^{-1}=\left(\begin{array}{cccccccc}
\frac{1}{2 N A_{0}} & \frac{1}{N A_{0}} & \frac{1}{N A_{0}} & \cdots & \frac{1}{N A_{0}} & \frac{1}{2 N A_{0}} \\
\frac{1}{N A_{1}} & \frac{2}{N A_{1}} \cos \frac{1 \times \pi}{N} & \frac{2}{N A_{1}} \cos \frac{2 \times \pi}{N} & \cdots & \frac{2}{N A_{1}} \cos \frac{(N-1) \pi}{N} & -\frac{1}{N A_{1}} \\
\frac{1}{N A_{2}} & \frac{2}{N A_{2}} \cos \frac{1 \times 2 \pi}{N} & \frac{2}{N A_{2}} \cos \frac{2 \times 2 \pi}{N} & \cdots & \frac{2}{N A_{2}} \cos \frac{(N-1) \times 2 \pi}{N} & \frac{1}{N A_{2}} \\
\vdots & \vdots & \vdots & & \vdots & \vdots \\
\frac{1}{N A_{k}} & \frac{2}{N A_{k}} \cos \frac{1 \times k \pi}{N} & \frac{2}{N A_{k}} \cos \frac{2 \times k \pi}{N} & \cdots & \frac{2}{N A_{k}} \cos \frac{(N-1) k \pi}{N} & \frac{(-1)^{k}}{N A_{k}} \\
\vdots & \vdots & \vdots & & \vdots & \vdots \\
\frac{1}{2 N A_{N}} & -\frac{1}{N A_{N}} & \frac{1}{N A_{N}} & \cdots & \frac{(-1)^{N-1}}{N A_{N}} & \frac{(-1)^{N}}{2 N A_{N}}
\end{array}\right)
\end{aligned}
$$




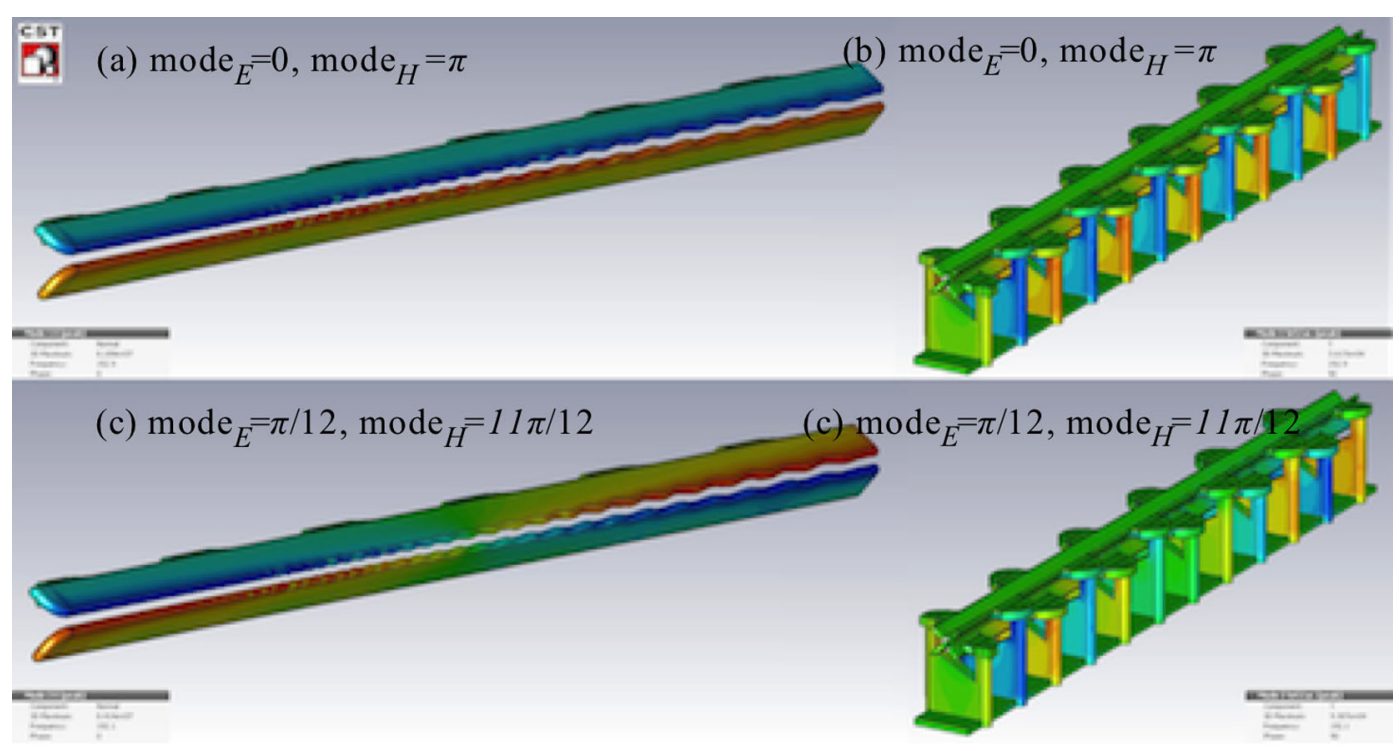

FIG. 16 (color online). The FNAL RFQ normal E-field component on the electrodes of the first two modes are shown in (a) and (c) and their corresponding currents on the stems are shown in (b) and (d). Both modes are quadrupole modes. The first mode has a constant E-field $(q=0)$ while the second mode shows one change in the field polarity $(q=1)$ along the structure. The quadrupole mode of the E-field is mode $_{E}=0$ in the fundamental state with the corresponding H-field having mode ${ }_{H}=\pi$. The highest quadrupole mode for the four-rod RFQ is mode $_{E}=\pi$ with mode $_{H}=0$ combination.

\section{APPENDIX C: MODES CALCULATED BY CST}

From our calculations discussed in Sec. II, we know that for $(N+1)$ tuning cells, there are $(N+1)$ possible modes. One way to number these modes is to look at the E-field phase shift per tuning cell

$$
\phi=q \pi / N,
$$

where $q=0,1, \ldots ., N$, as it can also be found in [18] for example. Here, we can interpret $q$ as the quantum number that tells us the number of cells where the E-field is zero, i.e. the number of nodes. We can identify the mode identities used in our paper, which are based on the current distribution and therefore are magnetic modes, i.e. $H$ modes. We will denote them as mode ${ }_{H}$ here. The relationship between $q$ and mode ${ }_{E}$ and $\operatorname{mode}_{H}$ is given below:

$$
\operatorname{mode}_{E}=\frac{q \pi}{N}, \quad \operatorname{mode}_{H}=\frac{(N-q) \pi}{N} .
$$

We illustrate the phase shift $\varphi$ using the FNAL RFQ as an example. We find that the lowest mode found by MWS is $152.9 \mathrm{MHz}$ and the next higher mode is $192.1 \mathrm{MHz}$, which are both quadrupole modes. For these two modes, the normal E-field components on the electrodes calculated by MWS are shown in Fig. 16(a) for $\operatorname{mode}_{E}=0$ and 16(c) with $\operatorname{mode}_{E}=1 \pi / 12$. The corresponding currents on the stems are shown in Figs. 16(b) and 16(d). As a comparison, we can see that for mode ${ }_{E}=0$, the current distributions flow in opposite directions between adjacent tuning cells corresponding to $\operatorname{mode}_{H}=\pi$ which is exactly like what we had described in Fig. 6. This fundamental mode of the fourrod RFQ is used as the operating mode and is called the $\pi-0$ mode.

The dispersion diagram up to $400 \mathrm{MHz}$ of the FNAL RFQ without any tuning is given in Fig. 17. It includes all modes simulated by MWS. Due to the connection of the electrodes to the stems, the higher order modes are far away from the fundamental mode, $q=0$, where the RFQ operates. The spectrum is grouped into transverse quadrupole modes (black squares) and the dipole and mixed modes (red dots). Sometimes, the dipole and higher order

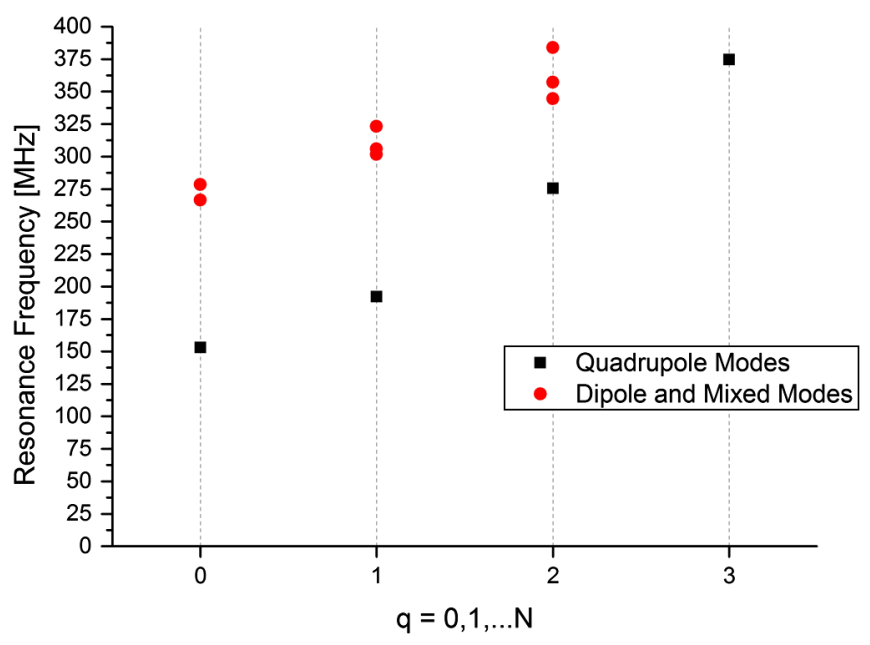

FIG. 17 (color online). The dispersion relation of the FNAL RFQ up to $400 \mathrm{MHz}$ calculated by MWS. The first two modes are quadrupole modes and the first dipolelike mode can be found at about $125 \mathrm{MHz}$ above the operating mode. 
transverse modes in the simulation results are hard to distinguish. In the four-rod RFQ, the fundamental mode of the E-field is always the quadrupole mode with a constant E-field $(q=0)$ along the structure. The next mode is also a quadrupole mode, but the potential on the electrodes changes sign once along the structure, a $q=1$ state. This mode is already $40 \mathrm{MHz}$ higher than the operating mode without any tuning. The first mode that shows a dipolelike potential distribution with a constant field along the structure is about $125 \mathrm{MHz}$ higher compared to the operating mode. Note, the weakness of our model mentioned in Sec. IIIB3 also manifests itself in predicting higher order eigenfrequencies that are too small.

[1] P. Fischer and A. Schempp, in Proceedings of the 10th European Particle Accelerator Conference, Edinburgh, Scotland, 2006 (EPS-AG, Edinburgh, Scotland, 2006), pp. 1583-1585.

[2] J. Schmidt et al., in Proceedings of the 3rd International Particle Accelerator Conference, New Orleans, Louisiana, USA, 2012, edited by J. Corbett et al. (IEEE, Piscataway, NJ, 2012), pp. 3818-3820.

[3] J. Schmidt et al., in Proceedings of Linac 2012, Tel-Aviv, Israel, 2012, pp. 963-965.

[4] H. Podlech et al., in Proceedings of the Particle Accelerator Conference, Chicago, IL, 2001 (IEEE, New York, 2001), pp. 3069-3071.

[5] S. Kurennoy, R. W. Gernett, and L. J. Rybarcyk, in Proceedings of IPAC 2013, Shanghai, China, pp. 3978-3980.
[6] A. Schempp et al., in Proceedings of LINAC 1984, Darmstadt, Germany, 1984, edited by U. Grundinger et al., pp. 100-102.

[7] V. Kapin, Jpn. J. Appl. Phys. 36, 2415 (1997).

[8] A. Palmieri et al., in Proceedings of the International Particle Accelerator Conference, Kyoto, Japan (ICR, Kyoto, 2010), pp. 606-608.

[9] J. X. Fang and A. Schempp, in Proceedings of EPAC 1992, Berlin, Germany, 1992, edited by J. Corbett et al., pp. 1331-1333.

[10] Intuitively, we can imagine that $\kappa_{1}$ and $\kappa_{2}$ can be different between cells even when the tuning plates are at the same height. However, when we look at Fig. 9, we see the MWS data can be well fitted with only two coupling constants $\kappa_{1}$ and $\kappa_{2}$.

[11] W. H. Hayt and J.E. Kemmerly, Engineering Circuit Analysis, 4th Edition (McGraw-Hill Book Company, New York, 1986).

[12] P. Fischer, Ph.D. thesis, Goethe University, Frankfurt am Main, 2007.

[13] C. Y. Tan et al., Technical Report No. Beams-doc-3646v16, 2013.

[14] K. Küpfmüller et al., Theoretische Elektrotechnik (Springer, New York, 2008).

[15] Some details about how, in general, the measurements are done can be found in Refs. [2,3,12]. The FNAL RFQ measurements are documented in [13], Sec. 4.3.4.

[16] P. Bevington and D. K. Robinson, Data Reduction and Error Analysis for the Physical Sciences (McGraw-Hill Book Company, New York, 2002).

[17] S. Kurennoy (private communication).

[18] M. Vretenar, in CAS-RF for Accelerators, Ebeltoft, Denmark, 2010. 